\title{
A binary encoding of spinors and applications
}

https://doi.org/10.1515/coma-2020-0100

Received April 22, 2020; accepted July 27, 2020

Abstract: We present a binary code for spinors and Clifford multiplication using non-negative integers and their binary expressions, which can be easily implemented in computer programs for explicit calculations. As applications, we present explicit descriptions of the triality automorphism of Spin(8), explicit representations of the Lie algebras $\mathfrak{s p i n}(8), \mathfrak{s p i n}(7)$ and $\mathfrak{g}_{2}$, etc.

Keywords: Spin representation, Clifford algebras, Spinor representation, octonions, triality

MSC: 15A66 15A69 53C27 17B10 17B25 20G41

\section{Introduction}

Spinors were first discovered by Cartan in 1913 [10], and have been of great relevance in Mathematics and Physics ever since. In this note, we introduce a binary code for spinors by using a suitable basis and setting up a correspondence between its elements and non-negative integers via their binary decompositions. Such a basis consists of weight vectors of the Spin representation as presented by Friedrich and Sulanke in 1979 [11] and which were originally described in a rather different and implicit manner by Brauer and Weyl in 1935 [6]. This basis is known to physiscits as a Fock basis [8]. The careful reader will notice that our (integer) encoding uses half the number of bits used by any other binary code of Clifford algebras [7, 18], thus making it computationally more efficient. Furthermore, Clifford multiplication of vectors with spinors (using the terminology of [12]) becomes a matter of flipping bits in binary expressions and keeping track of powers of $\sqrt{-1}$. In order to show its usefulness, we develop very explicit descriptions of some well-known facts such as the triality automorphism of Spin(8) (avoiding entirely any reference to the octonions), the relationship between Clifford multiplication and the multiplication table of the octonions, and the construction of sets of linearly independent vector fields on spheres. The natural binary/integer code of spinors, as well as the three applications, are the main contributions of the paper. The computer implementation of this code has allowed us to carry out many calculations in high dimensions which, otherwise, would have been impossible. Note that, although we only deal with the case of Clifford algebras and spinors defined by positive definite quadratic forms, the binary code can be easily extended to semi-definite quadratic forms.

The paper is organized as follows. In Section 2, we recall standard facts about Clifford algebras, the Spin Lie groups and algebras, the spinor representations and the basis of weight spinors. In Section 3, we introduce the correspondence between these basic spinors and nonnegative integers, as well as the functions that encode the Clifford multiplication. In Section 4, we present the aforementioned descriptions/applications: triality in dimension 8 , the relationship between Clifford multiplication and the multiplication table of the octonions and, finally, the construction of linearly independent vector fields on spheres.

Gerardo Arizmendi: Department of Actuarial Sciences, Physics and Mathematics, UDLAP, San Andrés Cholula, Puebla, México, E-mail: gerardo.arizmendi@udlap.mx

*Corresponding Author: Rafael Herrera: Centro de Investigación en Matemáticas, A. P. 402, 36000 Guanajuato, Guanajuato, México, E-mail: rherrera@cimat.mx 


\section{Preliminaries}

The details of the facts mentioned in this section can be found in $[5,12]$.

\subsection{Clifford algebra, spinors, Clifford multiplication and the Spin group}

Let $C l_{n}$ denote the Clifford algebra generated by all the products of the canonical vectors $e_{1}, e_{2}, \ldots, e_{n} \in \mathbb{R}^{n}$ subject to the relations

$$
\begin{aligned}
e_{j} e_{k}+e_{k} e_{j} & =-\left\langle e_{j}, e_{k}\right\rangle \text { for } j \neq k, \\
e_{j} e_{j} & =-1,
\end{aligned}
$$

where $\langle$,$\rangle denotes the standard inner product in \mathbb{R}^{n}$, and $C l_{n}^{0}$ the even Clifford subalgebra determined as the fixed point of the involution of $C l_{n}$ induced by $-\operatorname{Id}_{\mathbb{R}^{n}}$. Let

$$
\mathbb{C} l_{n}=C l_{n} \otimes_{\mathbb{R}} \mathbb{C}, \quad \text { and } \quad \mathbb{C l} l_{n}^{0}=C l_{n}^{0} \otimes_{\mathbb{R}} \mathbb{C}
$$

be the complexifications of $C l_{n}$ and $C l_{n}^{0}$. It is well known that

$$
\mathbb{C} l_{n} \cong\left\{\begin{array}{ll}
\operatorname{End}\left(\mathbb{C}^{2^{k}}\right), & \text { if } n=2 k, \\
\operatorname{End}\left(\mathbb{C}^{2^{k}}\right) \oplus \operatorname{End}\left(\mathbb{C}^{2^{k}}\right), & \text { if } n=2 k+1,
\end{array},\right.
$$

where

$$
\mathbb{C}^{2^{k}}=\underbrace{\mathbb{C}^{2} \otimes \ldots \otimes \mathbb{C}^{2}}_{k \text { times }}
$$

is the tensor product of $k=\left[\frac{n}{2}\right]$ copies of $\mathbb{C}^{2}$. Let us denote this space as

$$
\Delta_{n}=\mathbb{C}^{2^{k}},
$$

which is called the space of spinors. Consider the linear map

$$
\kappa: \mathbb{C} l_{n} \longrightarrow \operatorname{End}\left(\mathbb{C}^{2^{k}}\right),
$$

which is the aforementioned isomorphism for $n$ even, and the projection onto the first summand for $n$ odd.

The Spin group $\operatorname{Spin}(n) \subset C l_{n}$ is the subset

$$
\operatorname{Spin}(n)=\left\{x_{1} x_{2} \cdots x_{2 l-1} x_{2 l}\left|x_{j} \in \mathbb{R}^{n},\right| x_{j} \mid=1, l \in \mathbb{N}\right\},
$$

endowed with the product of the Clifford algebra. It is a Lie group and its Lie algebra is

$$
\mathfrak{s p i n}(n)=\operatorname{span}\left\{e_{i} e_{j} \mid 1 \leq i<j \leq n\right\} .
$$

Recall that the Spin group $\operatorname{Spin}(n)$ is the universal double cover of $S O(n), n \geq 3$. For $n=2$ we consider $\operatorname{Spin}(2)$ to be the connected double cover of $S O(2)$. The covering map will be denoted by

$$
\lambda_{n}: \operatorname{Spin}(n) \rightarrow \operatorname{SO}(n),
$$

where an element $x_{1} x_{2} \cdots x_{2 l-1} x_{2 l} \in \operatorname{Spin}(n)$ is mapped to the orthogonal transformation

$$
\begin{aligned}
\lambda_{n}: \mathbb{R}^{n} & \longrightarrow \mathbb{R}^{n} \\
y & \mapsto x_{1} x_{2} \cdots x_{2 l-1} x_{2 l} \cdot y \cdot x_{2 l} x_{2 l-1} \cdots x_{2} x_{1} .
\end{aligned}
$$

Its differential is given by

$$
\lambda_{n^{\star}}\left(e_{i} e_{j}\right)=2 E_{i j}
$$


where $E_{i j}=e_{i}^{\star} \otimes e_{j}-e_{j}^{\star} \otimes e_{i}$ is the standard basis of the skew-symmetric matrices and $e^{\star}$ denotes the metric dual of the vector $e$. We will also denote by $\lambda_{n}$ the induced representation on $\bigwedge^{\star} \mathbb{R}^{n}$.

The restriction of $\kappa$ to $\operatorname{Spin}(n)$ defines the Lie group representation

$$
\kappa_{n}: \operatorname{Spin}(n) \longrightarrow G L\left(\Delta_{n}\right),
$$

which is special unitary. We have the corresponding Lie algebra representation

$$
\kappa_{n^{\star}}: \mathfrak{s p i n}(n) \longrightarrow \mathfrak{g l}\left(\Delta_{n}\right),
$$

which is, in fact, the restriction of the linear map $\kappa: \mathbb{C} l_{n} \longrightarrow \operatorname{End}\left(\Delta_{\mathrm{n}}\right)$ to $\mathfrak{s p i n}(n) \subset \mathbb{C} l_{n}$. Note that $S O(1)=\{1\}$, $\operatorname{Spin}(1)=\{ \pm 1\}$, and $\Delta_{1}=\mathbb{C}$ the trivial 1-dimensional representation.

The Clifford multiplication is defined by

$$
\begin{aligned}
\mu_{n}: \mathbb{R}^{n} \otimes \Delta_{n} & \longrightarrow \Delta_{n} \\
x \otimes \psi & \mapsto \mu_{n}(x \otimes \psi)=x \cdot \psi:=\kappa(x)(\psi) .
\end{aligned}
$$

It is skew-symmetric with respect to the Hermitian product

$$
\left\langle x \cdot \psi_{1}, \psi_{2}\right\rangle=-\left\langle\psi_{1}, x \cdot \psi_{2}\right\rangle,
$$

is $\operatorname{Spin}(n)$-equivariant and can be extended to a $\operatorname{Spin}(n)$-equivariant map

$$
\begin{aligned}
\mu_{n}: \bigwedge^{*}\left(\mathbb{R}^{n}\right) \otimes \Delta_{n} & \longrightarrow \Delta_{n} \\
\omega \otimes \psi & \mapsto \omega \cdot \psi .
\end{aligned}
$$

Let

$$
\operatorname{vol}_{n}:=e_{1} e_{2} \cdots e_{n} .
$$

When $n$ is even, we define the following involution

$$
\begin{aligned}
\Delta_{n} & \longrightarrow \Delta_{n} \\
\psi & \mapsto(-i)^{\frac{n}{2}} \operatorname{Vol}_{n} \cdot \psi .
\end{aligned}
$$

The \pm 1 eigenspaces of this involution are denoted by $\Delta_{n}^{ \pm}$and called positive and negative spinors respectively. These spaces have equal dimension and are irreducible representations of $\operatorname{Spin}(n)$

$$
\kappa_{n}^{ \pm}: \operatorname{Spin}(n) \longrightarrow \operatorname{Aut}\left(\Delta_{n}^{ \pm}\right) .
$$

Note that our definition differs from the one given in [12] by a factor $(-1)^{\frac{n}{2}}$.

There exist either real or quaternionic structures on the spin representations. A quaternionic structure $\alpha$ on $\mathbb{C}^{2}$ is given by

$$
\alpha\left(\begin{array}{l}
z_{1} \\
z_{2}
\end{array}\right)=\left(\begin{array}{c}
-\bar{z}_{2} \\
\bar{z}_{1}
\end{array}\right)
$$

and a real structure $\beta$ on $\mathbb{C}^{2}$ is given by

$$
\beta\left(\begin{array}{l}
z_{1} \\
z_{2}
\end{array}\right)=\left(\begin{array}{c}
\bar{z}_{1} \\
\bar{z}_{2}
\end{array}\right)
$$

Note that these structures satisfy

$$
\begin{array}{ll}
\langle\alpha(v), w\rangle=\overline{\langle v, \alpha(w)\rangle}, & \langle\alpha(v), \alpha(w)\rangle=\overline{\langle v, w\rangle}, \\
\langle\beta(v), w\rangle=\overline{\langle v, \beta(w)\rangle}, & \langle\beta(v), \beta(w)\rangle=\overline{\langle v, w\rangle},
\end{array}
$$


with respect to the standard hermitian product in $\mathbb{C}^{2}$, where $v, w \in \mathbb{C}^{2}$. The real and quaternionic structures $\gamma_{n}$ on $\Delta_{n}=\left(\mathbb{C}^{2}\right)^{\otimes[n / 2]}$ are built as follows

$$
\begin{array}{lll}
\gamma_{n}=(\alpha \otimes \beta)^{\otimes 2 k} & \text { if } n=8 k, 8 k+1 & \text { (real), } \\
\gamma_{n}=(\alpha \otimes \beta)^{\otimes 2 k} \otimes \alpha & \text { if } n=8 k+2,8 k+3 & \text { (quaternionic), } \\
\gamma_{n}=(\alpha \otimes \beta)^{\otimes 2 k+1} & \text { if } n=8 k+4,8 k+5 & \text { (quaternionic), } \\
\gamma_{n}=(\alpha \otimes \beta)^{\otimes 2 k+1} \otimes \alpha & \text { if } n=8 k+6,8 k+7 & \text { (real). }
\end{array}
$$

which also satisfy

$$
\left\langle\gamma_{n}(v), w\right\rangle=\overline{\left\langle v, \gamma_{n}(w)\right\rangle}, \quad\left\langle\gamma_{n}(v), \gamma_{n}(w)\right\rangle=\overline{\langle v, w\rangle},
$$

where $v, w \in \Delta_{n}$. This means

$$
\left\langle v+\gamma_{n}(v), w+\gamma_{n}(w)\right\rangle \in \mathbb{R} .
$$

Now, we summarize some results about real representations of $C l_{r}^{0}$ in the next table (cf. [16]). Here $d_{r}$ denotes the dimension of an irreducible representation of $C l_{r}^{0}$ and $v_{r}$ the number of distinct irreducible representations.

\begin{tabular}{|c|c|c|c|}
\hline$r(\bmod 8)$ & $C l_{r}^{0}$ & $d_{r}$ & $v_{r}$ \\
\hline 1 & $\mathbb{R}\left(d_{r}\right)$ & $2^{\left\lfloor\frac{r}{2}\right\rfloor}$ & 1 \\
\hline 2 & $\mathbb{C}\left(d_{r} / 2\right)$ & $2^{\frac{r}{2}}$ & 1 \\
\hline 3 & $\mathbb{H}\left(d_{r} / 4\right)$ & $2^{\left\lfloor\frac{r}{2}\right\rfloor+1}$ & 1 \\
\hline 4 & $\mathbb{H}\left(d_{r} / 4\right) \oplus \mathbb{H}\left(d_{r} / 4\right)$ & $2^{\frac{r}{2}}$ & 2 \\
\hline 5 & $\mathbb{H}\left(d_{r} / 4\right)$ & $2^{\left\lfloor\frac{r}{2}\right\rfloor+1}$ & 1 \\
\hline 6 & $\mathbb{C}\left(d_{r} / 2\right)$ & $2^{\frac{r}{2}}$ & 1 \\
\hline 7 & $\mathbb{R}\left(d_{r}\right)$ & $2^{\left\lfloor\frac{r}{2}\right\rfloor}$ & 1 \\
\hline 8 & $\mathbb{R}\left(d_{r}\right) \oplus \mathbb{R}\left(d_{r}\right)$ & $2^{\frac{r}{2}-1}$ & 2 \\
\hline
\end{tabular}

Table 1

Let $\tilde{\Delta}_{r}$ denote the real irreducible representation of $C l_{r}^{0}$ for $r \not \equiv 0(\bmod 4)$ and $\tilde{\Delta}_{r}^{ \pm}$denote the real irreducible representations for $r \equiv 0(\bmod 4)$. Note that the representations are complex for $r \equiv 2,6(\bmod 8)$ and quaternionic for $r \equiv 3,4,5(\bmod 8)$.

\subsection{A special basis of spinors and an explicit description of $\kappa$}

In this subsection, we recall the explicit descriptions of $\kappa$ from [12] and the basis of spinors from [11], which were first discussed by Brauer and Weyl in [6].

The vectors

$$
u_{+1}=\frac{1}{\sqrt{2}}(1,-i) \quad \text { and } \quad u_{-1}=\frac{1}{\sqrt{2}}(1, i),
$$

form a unitary basis of $\mathbb{C}^{2}$. Consequently, the vectors

$$
\left\{u_{\left(\varepsilon_{1}, \ldots, \varepsilon_{k}\right)}=u_{\varepsilon_{1}} \otimes \ldots \otimes u_{\varepsilon_{k}} \mid \varepsilon_{j}= \pm 1, j=1, \ldots, k\right\},
$$

form a unitary basis of $\Delta_{n}=\left(\mathbb{C}^{2}\right)^{\otimes[n / 2]}$, which are known to be weight vectors of the Spin representation (see below).

In order to give an explicit description of the map $\kappa$, consider the following matrices with complex entries

$$
I d=\left(\begin{array}{ll}
1 & 0 \\
0 & 1
\end{array}\right), \quad g_{1}=\left(\begin{array}{cc}
i & 0 \\
0 & -i
\end{array}\right), \quad g_{2}=\left(\begin{array}{cc}
0 & i \\
i & 0
\end{array}\right), \quad T=\left(\begin{array}{cc}
0 & -i \\
i & 0
\end{array}\right) .
$$

Note that

$$
g_{1}\left(u_{ \pm 1}\right)=i u_{\mp 1}, \quad g_{2}\left(u_{ \pm 1}\right)= \pm u_{\mp 1}, \quad T\left(u_{ \pm 1}\right)=\mp u_{ \pm 1} .
$$


In general, the generators $e_{1}, \ldots, e_{n}$ of the Clifford algebra are mapped under $\kappa$ to the following linear transformations of $\Delta_{n}$ :

$$
\begin{aligned}
e_{1} & \mapsto I d \otimes I d \otimes \ldots \otimes I d \otimes I d \otimes g_{1}, \\
e_{2} & \mapsto I d \otimes I d \otimes \ldots \otimes I d \otimes I d \otimes g_{2}, \\
e_{3} & \mapsto I d \otimes I d \otimes \ldots \otimes I d \otimes g_{1} \otimes T, \\
e_{4} & \mapsto I d \otimes I d \otimes \ldots \otimes I d \otimes g_{2} \otimes T, \\
\vdots & \vdots \\
e_{2 k-1} & \mapsto g_{1} \otimes T \otimes \ldots \otimes T \otimes T \otimes T, \\
e_{2 k} & \mapsto g_{2} \otimes T \otimes \ldots \otimes T \otimes T \otimes T,
\end{aligned}
$$

and the last generator

$$
e_{2 k+1} \mapsto i T \otimes T \otimes \ldots \otimes T \otimes T \otimes T
$$

if $n=2 k+1$. Thus, if $1 \leq j \leq k$,

$$
\begin{aligned}
e_{2 j-1} u_{\varepsilon_{1}, \ldots, \varepsilon_{k}} & =i(-1)^{j-1}\left(\prod_{\alpha=k-j+2}^{k} \varepsilon_{\alpha}\right) u_{\varepsilon_{1}, \ldots,\left(-\varepsilon_{k-j+1}\right), \ldots, \varepsilon_{k}} \\
e_{2 j} u_{\varepsilon_{1}, \ldots, \varepsilon_{k}} & =(-1)^{j-1}\left(\prod_{\alpha=k-j+1}^{k} \varepsilon_{\alpha}\right) u_{\varepsilon_{1}, \ldots,\left(-\varepsilon_{k-j+1}\right), \ldots, \varepsilon_{k}}
\end{aligned}
$$

and

$$
e_{2 k+1} u_{\varepsilon_{1}, \ldots, \varepsilon_{k}}=i(-1)^{k}\left(\prod_{\alpha=1}^{k} \varepsilon_{\alpha}\right) u_{\varepsilon_{1}, \ldots, \varepsilon_{k}}
$$

if $n=2 k+1$ is odd. Also, the real and quaternionic structures on $\mathbb{C}^{2}$ look as follows

$$
\begin{aligned}
& \alpha\left(u_{\varepsilon}\right)=-\varepsilon i u_{-\varepsilon} \\
& \beta\left(u_{\varepsilon}\right)=u_{-\varepsilon},
\end{aligned}
$$

and, on the basis vectors of $\Delta_{n}$,

$$
\begin{aligned}
(\alpha \otimes \beta)^{\otimes k}\left(u_{\left(\varepsilon_{1}, \ldots, \varepsilon_{2 k}\right)}\right) & (-i)^{k}\left(\prod_{s=1}^{k} \varepsilon_{2 s-1}\right) u_{\left(-\varepsilon_{1}, \ldots,-\varepsilon_{2 k}\right)} \\
{\left[(\alpha \otimes \beta)^{\otimes k} \otimes \alpha\right]\left(u_{\left(\varepsilon_{1}, \ldots, \varepsilon_{2 k+1}\right)}\right) } & =(-i)^{k+1}\left(\prod_{s=1}^{k+1} \varepsilon_{2 s-1}\right) u_{\left(-\varepsilon_{1}, \ldots,-\varepsilon_{2 k+1}\right)}
\end{aligned}
$$

Remark. From these expressions, we can see that Clifford multiplication by basic vectors amounts to flipping a sign and keeping track of a power of $i=\sqrt{-1}$.

Remark. We became aware of these unitary bases in [5] and, since then, we have carried out many calculations in many dimensions. In spite of their usefulness, they are difficult to handle as tensor products in computer programs since they lead to large (sparse) matrices. Soon enough, we realized that we could encode such spinors and Clifford multiplication with binary expression (see Section 3), and perform calculations in higher dimensions which otherwise would have been impossible.

Example. In order to visualize the type of linear transformations given by $\kappa$, let us consider $n=6$ and the ordered spinor basis

$$
\left\{u_{(1,1,1)}, u_{(1,1,-1)}, u_{(1,-1,1)}, u_{(1,-1,-1)}, u_{(-1,1,1)}, u_{(-1,1,-1)}, u_{(-1,-1,1)}, u_{(-1,-1,-1)}\right\} .
$$


We have, for instance,

$$
\kappa_{6}\left(e_{1}\right)=\left(\begin{array}{cccccccc}
0 & i & & & & & & \\
i & 0 & & & & & & \\
& & 0 & i & & & & \\
& & i & 0 & & & & \\
& & & & 0 & i & & \\
& & & & i & 0 & & \\
& & & & & & 0 & i \\
& & & & & & i & 0
\end{array}\right), \quad \kappa_{6}\left(e_{2}\right)=\left(\begin{array}{cccccccc}
0 & -1 & & & & & & \\
1 & 0 & & & & & & \\
& & 0 & -1 & & & & \\
& & 1 & 0 & & & & \\
& & & & 0 & -1 & & \\
& & & 1 & 0 & & \\
& & & & & 0 & -1 \\
& & & & & & 1 & 0
\end{array}\right) .
$$

\subsubsection{Maximal torus of $\operatorname{Spin}(n)$ and weight vectors of $\Delta_{n}$}

A maximal torus of the group $\operatorname{Spin}(n)$ is given by

$$
\left\{\prod_{i=1}^{[n / 2]}\left(\cos \left(\theta_{i}\right)+\sin \left(\theta_{i}\right) e_{2 i-1} e_{2 i}\right) \in \operatorname{Spin}(n): \theta_{i} \in[0,2 \pi], i=1, \ldots,[n / 2]\right\} .
$$

In order to give a clear idea of the Spin representation, and not for computational purposes, we will write down some matrices corresponding to the transformations of $\Delta_{n}$ given by $\kappa_{n}\left(e_{i} e_{j}\right)$ and $\lambda_{n}\left(e_{i} e_{j}\right)$, for $n=6$ and some $1 \leq i<j \leq 6$. First consider the element $e_{1} e_{2} \in \operatorname{Spin}(6)$. In terms of (1) and (2)

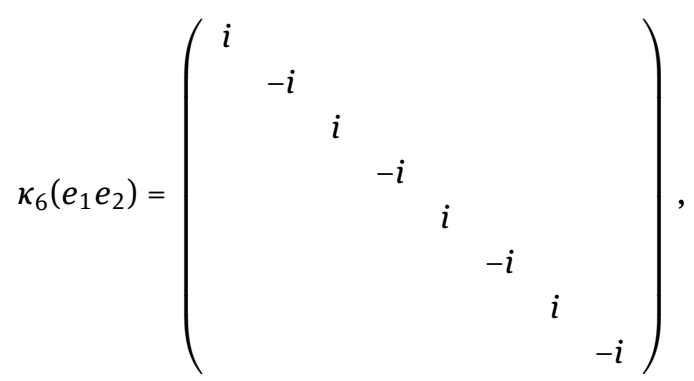

On the other hand, $e_{1} e_{2}$ acts on $y \in \mathbb{R}^{6}$ as follows

$$
\begin{aligned}
e_{1} e_{2}\left(y_{1} e_{1}+y_{2} e_{2}+y_{3} e_{3}+y_{4} e_{4}+y_{5} e_{5}+y_{6} e_{6}\right) e_{2} e_{1} & =e_{1}\left(y_{1} e_{1}-y_{2} e_{2}+y_{3} e_{3}+y_{4} e_{4}+y_{5} e_{5}+y_{6} e_{6}\right) e_{1} \\
& =-y_{1} e_{1}-y_{2} e_{2}+y_{3} e_{3}+y_{4} e_{4}+y_{5} e_{5}+y_{6} e_{6}
\end{aligned}
$$

i.e.

$$
\lambda_{6}\left(e_{1} e_{2}\right)=\left(\begin{array}{cccccc}
-1 & & & & & \\
& -1 & & & & \\
& & 1 & & & \\
& & & 1 & & \\
& & & & 1 & \\
& & & & & 1
\end{array}\right)
$$

Now, consider the element

$$
\cos (\theta)+\sin (\theta) e_{1} e_{2}=\left(\cos (\theta) e_{1}+\sin (\theta) e_{2}\right)\left(-e_{1}\right) \in \operatorname{Spin}(6) .
$$


On the one hand,

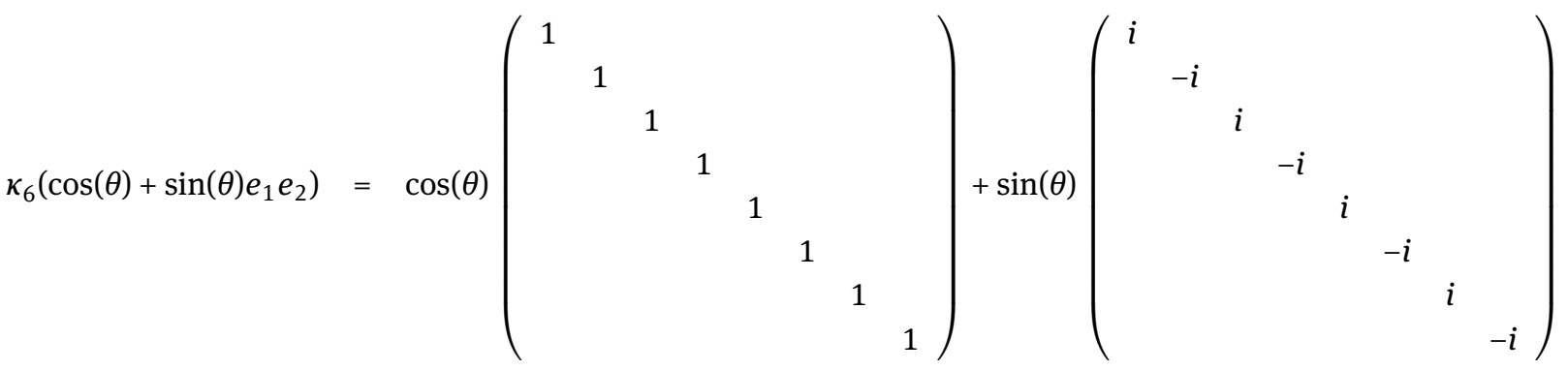

$$
\begin{aligned}
& =\left(\begin{array}{ccccccc}
e^{i \theta} & & & & & & \\
\\
& e^{-i \theta} & & & & & \\
& & e^{i \theta} & & & & \\
& & e^{-i \theta} & & & & \\
& & & e^{i \theta} & & & \\
& & & e^{-i \theta} & & \\
& & & & e^{i \theta} & \\
& & & & & e^{-i \theta}
\end{array}\right),
\end{aligned}
$$

and, on the other,

$$
\left(\cos (\theta) e_{1}+\sin (\theta) e_{2}\right)\left(-e_{1}\right) y\left(-e_{1}\right)\left(\cos (\theta) e_{1}+\sin (\theta) e_{2}\right)
$$

induces the transformation on $\mathbb{R}^{6}$

$$
\left(\begin{array}{cccccc}
\cos (2 \theta) & -\sin (2 \theta) & & & & \\
\sin (2 \theta) & \cos (2 \theta) & & & & \\
& & 1 & & & \\
& & 1 & & \\
& & & & 1 & \\
& & & & & 1
\end{array}\right) .
$$

Clearly, the two transformations $\kappa_{6}\left(\cos (\theta)+\sin (\theta) e_{1} e_{2}\right)$ and $\lambda_{6}\left(\cos (\theta)+\sin (\theta) e_{1} e_{2}\right)$ are different. Setting $\theta=\varphi_{1} / 2$, we see the familiar coefficients $\pm 1 / 2$ of the Spin representation

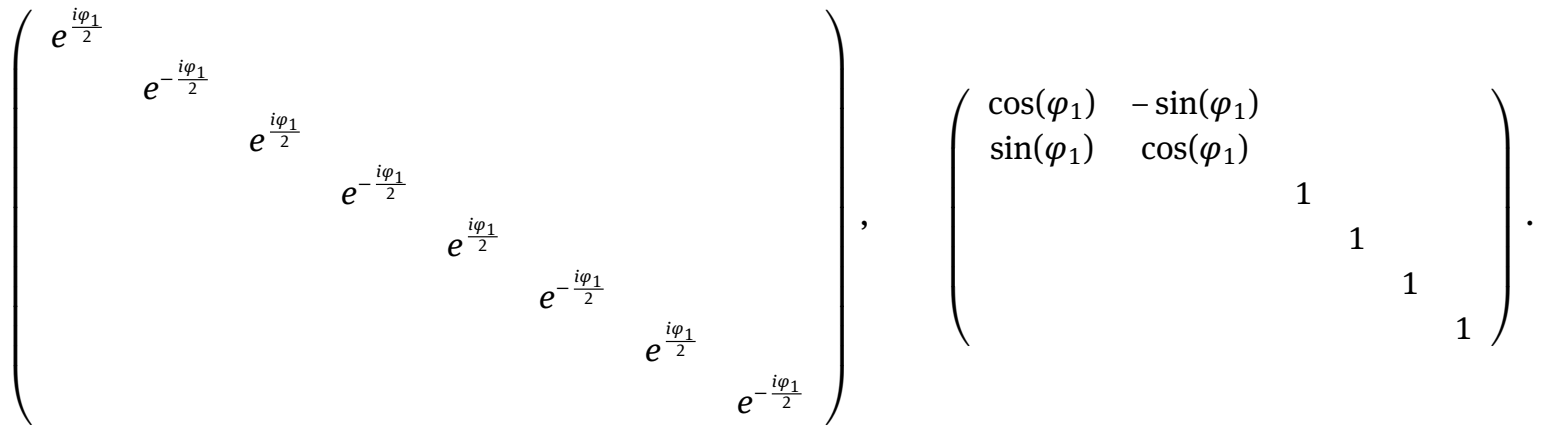

Similarly, $\cos \left(\varphi_{2} / 2\right)+\sin \left(\varphi_{2} / 2\right) e_{3} e_{4}$ induces

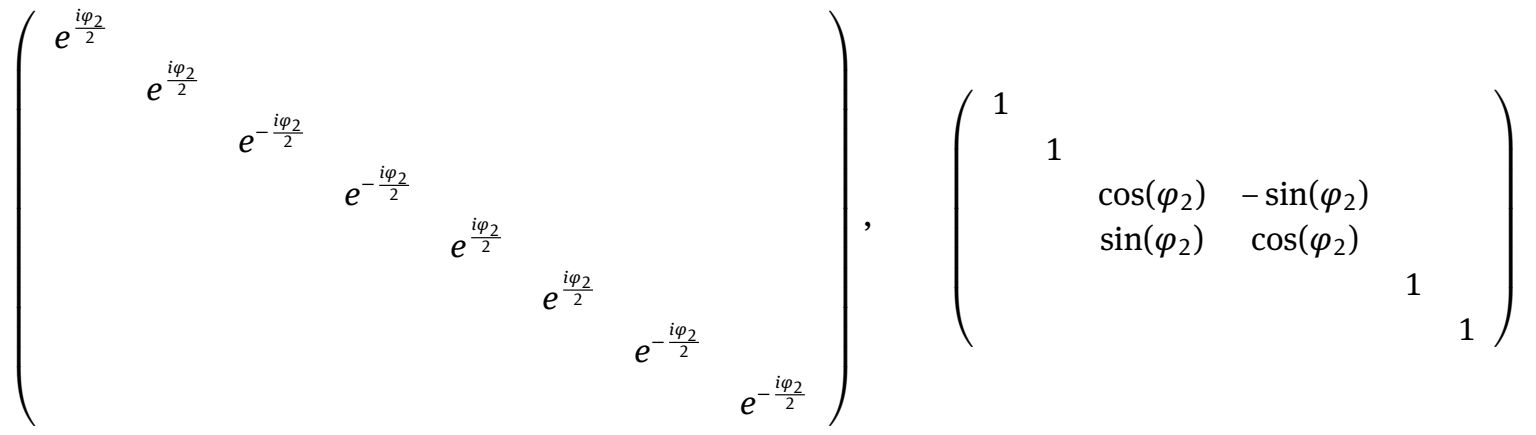


and $\cos \left(\varphi_{3} / 2\right)+\sin \left(\varphi_{3} / 2\right) e_{5} e_{6}$ induces

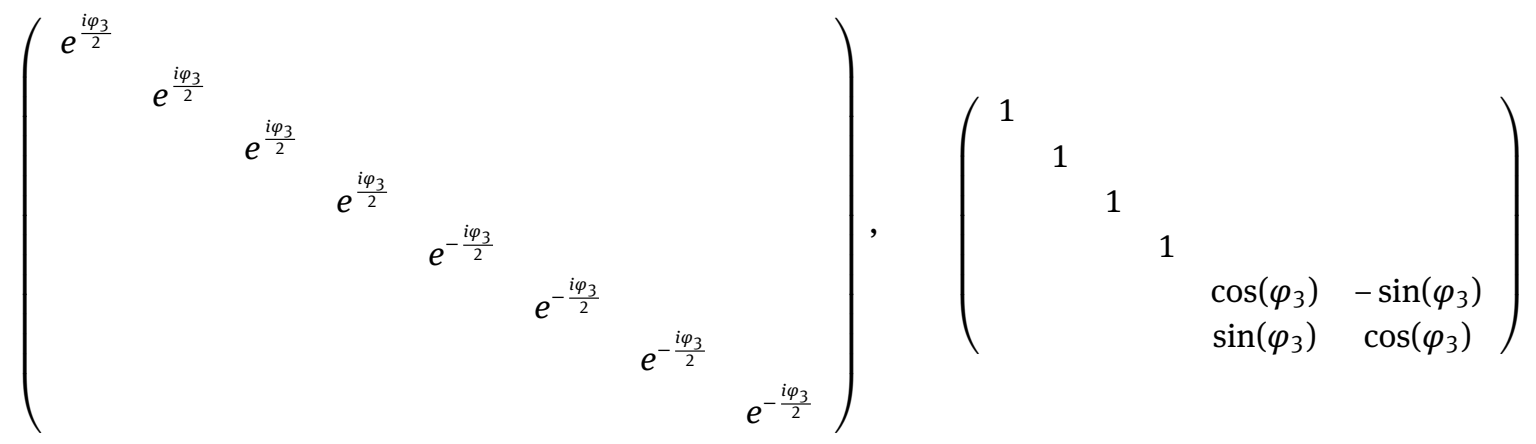

A general element of the standard maximal torus of $\operatorname{Spin}(6)$

$$
\left(\cos \left(\frac{\varphi_{1}}{2}\right)+\sin \left(\frac{\varphi_{1}}{2}\right) e_{1} e_{2}\right)\left(\cos \left(\frac{\varphi_{2}}{2}\right)+\sin \left(\frac{\varphi_{2}}{2}\right) e_{3} e_{4}\right)\left(\cos \left(\frac{\varphi_{3}}{2}\right)+\sin \left(\frac{\varphi_{3}}{2}\right) e_{5} e_{6}\right)
$$

has the following matrix representations

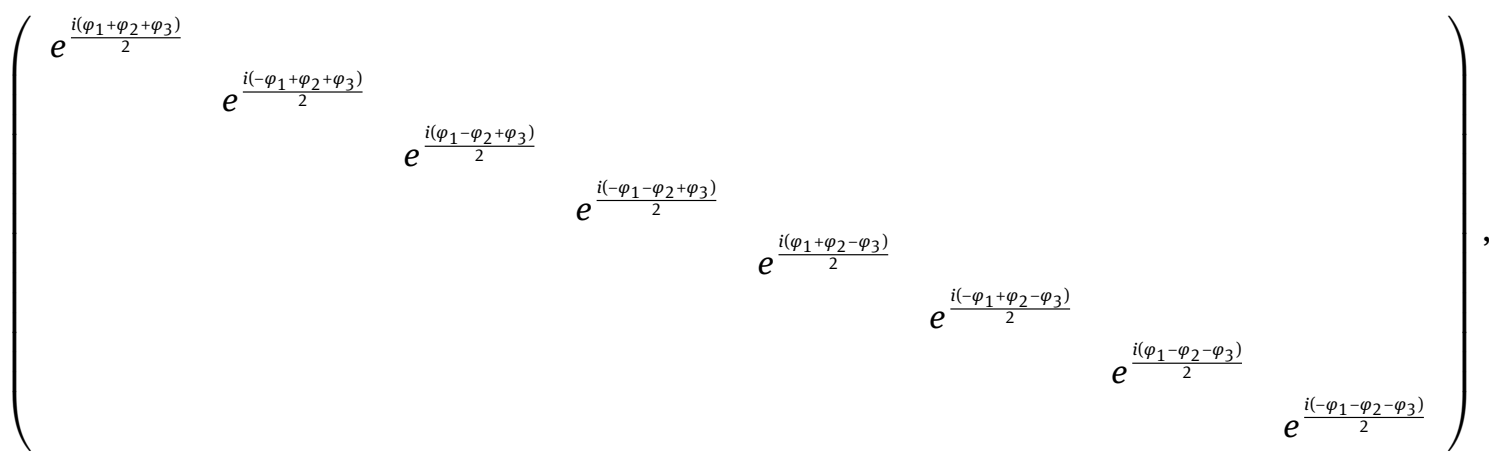

$$
\begin{aligned}
& \left(\begin{array}{cccccc}
\cos \left(\varphi_{1}\right) & -\sin \left(\varphi_{1}\right) & & & & \\
\sin \left(\varphi_{1}\right) & \cos \left(\varphi_{1}\right) & & & & \\
& & \cos \left(\varphi_{2}\right) & -\sin \left(\varphi_{2}\right) & & \\
& & \sin \left(\varphi_{2}\right) & \cos \left(\varphi_{2}\right) & & \\
& & & & \cos \left(\varphi_{3}\right) & -\sin \left(\varphi_{3}\right) \\
& & & & \sin \left(\varphi_{3}\right) & \cos \left(\varphi_{3}\right)
\end{array}\right) .
\end{aligned}
$$

This formula clearly shows that the basis given in (1) is made up of weight vectors of the spin representation $\Delta_{6}$. In general we have

$$
\prod_{i=1}^{[n / 2]}\left(\cos \left(\frac{\varphi_{i}}{2}\right)+\sin \left(\frac{\varphi_{i}}{2}\right) e_{2 i-1} e_{2 i}\right) \cdot u_{\left(\varepsilon_{1}, \ldots, \varepsilon_{k}\right)}=e^{\frac{i\left(\varepsilon_{1} \varphi_{1}+\ldots+\varepsilon_{k} \varphi_{k}\right)}{2}} u_{\left(\varepsilon_{1}, \ldots, \varepsilon_{k}\right)} .
$$

\section{Binary code}

Given the description in the previous section, we see that the calculation of $e_{j} u_{\varepsilon_{1}, \ldots, \varepsilon_{k}}$, where $k=[n / 2]$, depends on $j$, the $k$-tuple $\left(\varepsilon_{1}, \ldots, \varepsilon_{k}\right)$ and (possibly on) $n$. By noticing that

$$
+1=(-1)^{0} \text { and }-1=(-1)^{1},
$$

we see that for $\varepsilon= \pm 1$,

$$
\varepsilon=(-1)^{\frac{1-\varepsilon}{2}} .
$$

Thus, we can change the $k$-tuple $\left(\varepsilon_{1}, \ldots, \varepsilon_{k}\right)$ by the $k$-tuple $\left[\frac{1-\varepsilon_{1}}{2}, \ldots, \frac{1-\varepsilon_{k}}{2}\right]$ whose entries belong to $\{0,1\}$. Notice that these arrays correspond to the binary expressions of non-negative integers. For instance, for $n=6$, 


\begin{tabular}{|c|c|c|}
\hline$\left(\varepsilon_{1}, \varepsilon_{2}, \varepsilon_{3}\right)$ & {$\left[\frac{1-\varepsilon_{1}}{2}, \frac{1-\varepsilon_{2}}{2}, \frac{1-\varepsilon_{3}}{2}\right]$} & Integer \\
\hline$(1,1,1)$ & {$[0,0,0]$} & 0 \\
$(1,1,-1)$ & {$[0,0,1]$} & 1 \\
$(1,-1,1)$ & {$[0,1,0]$} & 2 \\
$(1,-1,-1)$ & {$[0,1,1]$} & 3 \\
$(-1,1,1)$ & {$[1,0,0]$} & 4 \\
$(-1,1,-1)$ & {$[1,0,1]$} & 5 \\
$(-1,-1,1)$ & {$[1,1,0]$} & 6 \\
$(-1,-1,-1)$ & {$[1,1,1]$} & 7 \\
\hline
\end{tabular}

Thus, the aforementioned binary code of spinors is given by the correspondence

$$
\left(\varepsilon_{1}, \ldots, \varepsilon_{k}\right) \longleftrightarrow \frac{1-\varepsilon_{k}}{2}\left(2^{0}\right)+\frac{1-\varepsilon_{k-1}}{2}\left(2^{1}\right)+\ldots+\frac{1-\varepsilon_{2}}{2}\left(2^{k-2}\right)+\frac{1-\varepsilon_{1}}{2}\left(2^{k-1}\right) .
$$

Remark. The careful reader will notice that for $n=2 k$ of $n=2 k+1$, this binary encoding of spinors uses $k$ bits as opposed to the $2 k$ bits of the binary encodings of Clifford algebras [18]. Since the classical descriptions of the space of spinors are given in terms of minimal ideals within the Clifford algebra itself, the inherited binary codes on such minimal ideals use twice as many bits as ours.

\subsection{Clifford multiplication}

The Clifford multiplication of a standard basis vectors $e_{p}$ with a spinors $u_{a}$, where $a \in\left\{0,1,2, \ldots, 2^{[n / 2]}-1\right\}$ now looks as follows

$$
\begin{aligned}
e_{2 j-1} u_{a} & =i(-1)^{j-1}(-1)^{\sum_{l=0}^{j-2}\left[a / 2^{l}\right]-2\left[a / 2^{l+1}\right]} u_{a+(-1)^{\left[a / 2^{j-1}-2\left[a / 2^{j}\right]\right.} 2^{j-1}} \\
e_{2 j} u_{a} & =(-1)^{j-1}(-1)^{\sum_{l=0}^{j-1}\left[a / 2^{l}\right]-2\left[a / 2^{l+1}\right]} u_{a+(-1)^{\left[a / 2^{j-1}\right]-2\left[a / 2^{j}\right]} 2_{2 j-1},}
\end{aligned}
$$

which can be summarized in one formula, for $1 \leq p \leq n, j:=\left[\frac{p+1}{2}\right]$,

$$
e_{p} u_{a}=(-1)^{2 j-p / 2-1+\sum_{l=0}^{j-2} a_{l}+a_{j-1}(-2 j+p+1)} u_{a+(-1)^{a_{j-1} 2^{j-1}}}
$$

where $a_{l}=\left[\frac{a}{2^{l}}\right]-2\left[\frac{a}{2^{l+1}}\right]$. Furthermore, if $n$ is odd, $k=\left[\frac{n}{2}\right]$,

$$
e_{2 k+1} u_{a}=i(-1)^{k+\sum_{l=0}^{k-1} a_{l}} u_{a} .
$$

These formulas allow us to make general assertions and perform computations in large dimensions without the use of enormous matrices (recall that the dimension of the $\operatorname{Spin}(n)$ representations increases exponentially with $n$ ).

Remark. This approach also has an important consequence: while formulas (3) and (4) seem to depend on $n$, once we write things down using integers in (5), it becomes apparent that Clifford multiplication does not depend on $n$ if $n$ is even. For instance, we will always have

$$
e_{5} u_{10}=i u_{15}
$$

for all $n \geq 6$. We can actually make the following (non-sharp) claim.

Proposition 3.1. Let $p \in \mathbb{N}$ and $a \in \mathbb{N} \cup\{0\}$. Formula (5) does not depend on $n$ if $n>\max \left\{p, 1+2 \log _{2}(a+1)\right\}$.

In this sense, formula (5) is rather universal, but still depends on standard inclusions of Euclidean spaces and their associated Clifford algebras, as well as the explicit mapping of generators (2). 


\subsubsection{Example: the isomorphism between $\Delta_{2 k-1}$ and $\Delta_{2 k}^{+}$}

The space of positive spinors $\Delta_{2 k}^{+}$is generated by the elements $u_{\varepsilon_{1} \cdots \varepsilon_{k}}$ such that

$$
\prod_{l=1}^{k} \varepsilon_{l}=1
$$

In the binary code this corresponds to the nonnegative integers whose binary expansion has an even number of bits $a_{l}=\left[\frac{a}{2^{l}}\right]-2\left[\frac{a}{2^{l+1}}\right]$ equal to 1 .

Now, the isomorphism

$$
f: \Delta_{2 k-1}=\operatorname{span}\left\{u_{a} \in \mathbb{Z} \mid 0 \leq a \leq 2^{k-1}-1\right\} \longrightarrow \Delta_{2 k}^{+}=\operatorname{span}\left\{u_{b} \in \mathbb{Z} \mid 0 \leq b \leq 2^{k}-1, \sum_{l=0}^{k-1} b_{l} \equiv 0(\bmod 2)\right\},
$$

as representations of the Lie algebra $\mathfrak{s p i n}(2 k-1)$, is given by

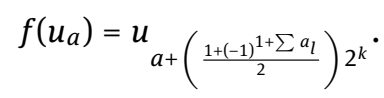

In order to check that the complex linear extension of $f$ is $\mathfrak{s p i n}(2 k-1)$ equivariant, let $0 \leq a \leq 2^{k-1}, 1 \leq p \leq$ $q \leq 2 k-1$. We must verify

$$
f\left(e_{p} e_{q}\left(u_{a}\right)\right)=e_{p} e_{q}\left(f\left(u_{a}\right)\right) .
$$

Note that the subindices of $u_{a}$ and $f\left(u_{a}\right)$ have the same binary expression up to the digit corresponding to $2^{k-1}$ so that for $1 \leq p<q \leq 2 k-2$ the identity (7) is fulfilled. The only cases we have to check are $1 \leq p \leq 2 k-2$ and $q=2 k-1$. On the one hand

$$
e_{2 k-1} u_{a}=i(-1)^{k-1}(-1)^{\sum_{l=0}^{k-2} a_{l}} u_{a}
$$

when $u_{a}$ is considered as a spinor in $\Delta_{2 k-1}$ and

$$
e_{2 k-1} u_{a}=i(-1)^{k-1}(-1)^{\sum_{l=0}^{k-2} a_{l}} u_{a+(-1)^{a_{k-1} 2^{k-1}}}
$$

when $u_{a}$ is considered as a spinor in $\Delta_{2 k}^{+}$.

On the other hand, in $\Delta_{2 k-1}$

$$
e_{p} e_{2 k-1} u_{a}=i(-1)^{k-1}(-1)^{\sum_{l=0}^{k-2} a_{l}}(-1)^{2 j-p / 2-1}(-1)^{\sum_{l=0}^{j-2} a_{l}}(-1)^{a_{j-1}(-2 j+p+1)} u_{a+(-1)^{a_{j-1}} 2^{j-1}} .
$$

Remark. One can even avoid the use of (6) when $n$ is odd and $p=n$ by using the isomorphism between $\Delta_{2 k-1}=\Delta_{2 k}^{+}$.

\section{Applications}

In this long section, we present three applications of the binary code in the form of explicit calculations of the following well-knoun facts: triality in dimension 8 without any reference to the octonions (compare with $[9,13,17,18]$ ), the octonion multiplication table (compare with $[4,17])$ and the construction of independent vector fields on spheres (compare with [20]).

\subsection{Triality}

We will first recall the idea of triality in a topological form. As we will recall below, the group Spin(8) is represented orthogonally on three real 8-dimensional spaces: $\mathbb{R}^{8}, \tilde{\Delta}_{8}^{+}$and $\tilde{\Delta}_{8}^{-}$. In other words, we have three 
homomorphisms

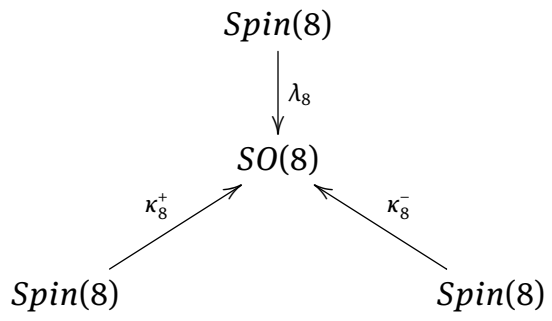

Now, consider the following two diagrams,
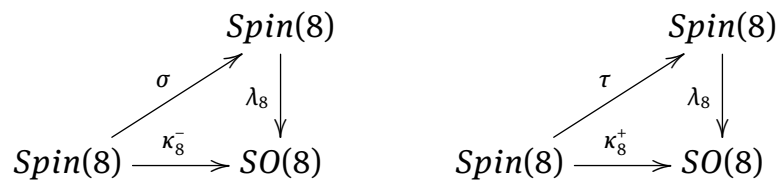

which include the correspoding lifts $\sigma$ and $\tau$ (due to the simple connectedness of Spin(8)). We will see that $\sigma$ : $\operatorname{Spin}(8) \longrightarrow \operatorname{Spin}(8)$ is an outer automorphism of order 3 (a triality automorphism), $\tau$ : Spin(8) $\longrightarrow \operatorname{Spin}(8)$ is an outer automorphism of order 2, and the two automorphisms generate a copy of the permutation group $S_{3}$.

First, we will examine the situation explicitly at the Lie algebra level
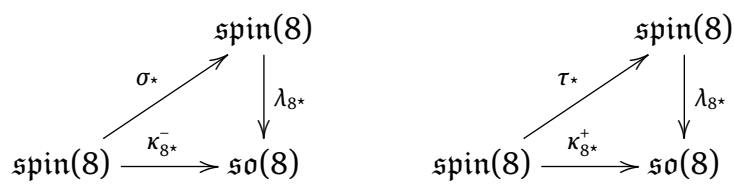

and later at the Lie group level.

\subsubsection{The real $\operatorname{Spin}(8)$-representations $\tilde{\Delta}_{8}^{+}$and $\tilde{\Delta}_{8}^{+}$}

Recall that $\gamma_{8}: \Delta_{8} \longrightarrow \Delta_{8}$ is a real structure on $\Delta_{8}$, which means it is the complexification of a real vector space $\tilde{\Delta}_{8}$ given by

$$
\tilde{\Delta}_{8}=\left(1+\gamma_{8}\right)\left(\Delta_{8}\right) \text {. }
$$

Furthermore, $\gamma_{8}$ also preserves the subrepresentations $\Delta_{8}^{+}$and $\Delta_{8}^{-}$, i.e. $\gamma_{8}$ restricts to real structures on $\Delta_{8}^{+}$and $\Delta_{8}^{-}$and, therefore, they are also complexifications of real vector spaces

$$
\begin{aligned}
\tilde{\Delta}_{8}^{+} & =\left\{(+1) \text {-eigenspace of } \gamma_{8} \text { in } \Delta_{8}^{+}\right\} \\
& =\left(1+\gamma_{8}\right)\left(\Delta_{8}^{+}\right), \\
\tilde{\Delta}_{8}^{-} & =\left\{(-1) \text {-eigenspace of } \gamma_{8} \text { in } \Delta_{8}^{-}\right\} \\
& =\left(1-\gamma_{8}\right)\left(\Delta_{8}^{-}\right) .
\end{aligned}
$$

In fact, we have chosen $\tilde{\Delta}_{8}^{+}$and $\tilde{\Delta}_{8}^{-}$in this way so that they are compatible with Clifford multiplication

$$
\mu_{8}: \mathbb{R}^{8} \times \tilde{\Delta}_{8}^{+} \longrightarrow \tilde{\Delta}_{8}^{-}
$$

We have explicit generators for the complex spinor spaces

$$
\begin{aligned}
& \Delta_{8}^{+}=\operatorname{span}\left(u_{0}, u_{3}, u_{5}, u_{6}, u_{9}, u_{10}, u_{12}, u_{15}\right), \\
& \Delta_{8}^{-}=\operatorname{span}\left(u_{1}, u_{2}, u_{4}, u_{7}, u_{8}, u_{11}, u_{13}, u_{14}\right) .
\end{aligned}
$$

For the real representation $\tilde{\Delta}_{8}^{+}$we have

$$
\tilde{\Delta}_{8}^{+}=\operatorname{span}\left\{\frac{1}{\sqrt{2}}\left(u_{0}-u_{15}\right), \frac{i}{\sqrt{2}}\left(u_{0}+u_{15}\right), \frac{1}{\sqrt{2}}\left(u_{3}+u_{12}\right), \frac{i}{\sqrt{2}}\left(u_{3}-u_{12}\right),\right.
$$




$$
\left.\frac{1}{\sqrt{2}}\left(u_{5}-u_{10}\right), \frac{i}{\sqrt{2}}\left(u_{5}+u_{10}\right), \frac{1}{\sqrt{2}}\left(u_{6}+u_{9}\right), \frac{i}{\sqrt{2}}\left(u_{6}-u_{9}\right)\right\} \text {. }
$$

We choose the ordered basis of $\tilde{\Delta}_{8}^{-}$to be the image of the basis of $\tilde{\Delta}_{8}^{+}$under Clifford multiplication by the canonical vector $e_{1} \in \mathbb{R}^{8}$. Namely,

$$
\begin{aligned}
\tilde{\Delta}_{8}^{-}= & \operatorname{span}\left\{\frac{i}{\sqrt{2}}\left(u_{1}-u_{14}\right), \frac{-1}{\sqrt{2}}\left(u_{1}+u_{14}\right), \frac{i}{\sqrt{2}}\left(u_{2}+u_{13}\right), \frac{1}{\sqrt{2}}\left(u_{2}-u_{13}\right),\right. \\
& \left.\frac{-i}{\sqrt{2}}\left(u_{4}-u_{11}\right), \frac{-1}{\sqrt{2}}\left(u_{4}+u_{11}\right), \frac{i}{\sqrt{2}}\left(u_{7}+u_{8}\right), \frac{-1}{\sqrt{2}}\left(u_{7}-u_{8}\right)\right\} .
\end{aligned}
$$

\subsubsection{The endomorphism $\sigma_{\star}$}

Using the ordered basis of spinors, one can compute the endomorphisms corresponding to the generators $e_{i} e_{j} \in \mathfrak{s p i n}(8), 1 \leq i<j \leq 8$, under the map $\kappa_{8^{\star}}^{-}$and, in turn, express those endomorphisms as images of elements of $\mathfrak{s p i n}(8)$ under $\lambda_{8^{\star}}$ :

$$
\begin{aligned}
& \kappa_{8^{\star}}^{-}\left(e_{1} e_{2}\right)=-E_{1,2}-E_{3,4}-E_{5,6}-E_{7,8}=\frac{1}{2} \lambda_{8^{\star}}\left(-e_{1} e_{2}-e_{3} e_{4}-e_{5} e_{6}-e_{7} e_{8}\right), \\
& \kappa_{8^{\star}}^{-}\left(e_{1} e_{3}\right)=-E_{1,3}+E_{2,4}-E_{5,7}+E_{6,8}=\frac{1}{2} \lambda_{8^{\star}}\left(-e_{1} e_{3}+e_{2} e_{4}-e_{5} e_{7}+e_{6} e_{8}\right) \text {, } \\
& \kappa_{8^{\star}}^{-}\left(e_{1} e_{4}\right)=-E_{1,4}-E_{2,3}+E_{5,8}+E_{6,7}=\frac{1}{2} \lambda_{8^{\star}}\left(-e_{1} e_{4}-e_{2} e_{3}+e_{5} e_{8}+e_{6} e_{7}\right) \text {, } \\
& \kappa_{8^{\star}}^{-}\left(e_{1} e_{5}\right)=-E_{1,5}+E_{2,6}+E_{3,7}-E_{4,8}=\frac{1}{2} \lambda_{8^{\star}}\left(-e_{1} e_{5}+e_{2} e_{6}+e_{3} e_{7}-e_{4} e_{8}\right) \text {, } \\
& \kappa_{8^{\star}}^{-}\left(e_{1} e_{6}\right)=-E_{1,6}-E_{2,5}-E_{3,8}-E_{4,7}=\frac{1}{2} \lambda_{8^{\star}}\left(-e_{1} e_{6}-e_{2} e_{5}-e_{3} e_{8}-e_{4} e_{7}\right) \text {, } \\
& \kappa_{8^{\star}}^{-}\left(e_{1} e_{7}\right)=-E_{1,7}+E_{2,8}-E_{3,5}+E_{4,6}=\frac{1}{2} \lambda_{8^{\star}}\left(-e_{1} e_{7}+e_{2} e_{8}-e_{3} e_{5}+e_{4} e_{6}\right) \text {, } \\
& \kappa_{8^{\star}}^{-}\left(e_{1} e_{8}\right)=-E_{1,8}-E_{2,7}+E_{3,6}+E_{4,5}=\frac{1}{2} \lambda_{8^{\star}}\left(-e_{1} e_{8}-e_{2} e_{7}+e_{3} e_{6}+e_{4} e_{5}\right) \text {, } \\
& \kappa_{8^{\star}}^{-}\left(e_{2} e_{3}\right)=E_{1,4}+E_{2,3}+E_{5,8}+E_{6,7}=\frac{1}{2} \lambda_{8^{\star}}\left(e_{1} e_{4}+e_{2} e_{3}+e_{5} e_{8}+e_{6} e_{7}\right) \text {, } \\
& \kappa_{8^{\star}}^{-}\left(e_{2} e_{4}\right)=-E_{1,3}+E_{2,4}+E_{5,7}-E_{6,8}=\frac{1}{2} \lambda_{8^{\star}}\left(e_{1} e_{4}+e_{2} e_{3}+e_{5} e_{8}+e_{6} e_{7}\right) \text {, } \\
& \kappa_{8^{\star}}^{-}\left(e_{2} e_{5}\right)=E_{1,6}+E_{2,5}-E_{3,8}-E_{4,7}=\frac{1}{2} \lambda_{8^{\star}}\left(e_{1} e_{6}+e_{2} e_{5}-e_{3} e_{8}-e_{4} e_{7}\right) \text {, } \\
& \kappa_{8^{\star}}^{-}\left(e_{2} e_{6}\right)=-E_{1,5}+E_{2,6}-E_{3,7}+E_{4,8}=\frac{1}{2} \lambda_{8^{\star}}\left(-e_{1} e_{5}+e_{2} e_{6}-e_{3} e_{7}+e_{4} e_{8}\right) \text {, } \\
& \kappa_{8^{\star}}^{-}\left(e_{2} e_{7}\right)=E_{1,8}+E_{2,7}+E_{3,6}+E_{4,5}=\frac{1}{2} \lambda_{8^{\star}}\left(e_{1} e_{8}+e_{2} e_{7}+e_{3} e_{6}+e_{4} e_{5}\right) \text {, } \\
& \kappa_{8^{\star}}^{-}\left(e_{2} e_{8}\right)=-E_{1,7}+E_{2,8}+E_{3,5}-E_{4,6}=\frac{1}{2} \lambda_{8^{\star}}\left(-e_{1} e_{7}+e_{2} e_{8}+e_{3} e_{5}-e_{4} e_{6}\right) \text {, } \\
& \kappa_{8^{\star}}^{-}\left(e_{3} e_{4}\right)=E_{1,2}+E_{3,4}-E_{5,6}-E_{7,8}=\frac{1}{2} \lambda_{8^{\star}}\left(e_{1} e_{2}+e_{3} e_{4}-e_{5} e_{6}-e_{7} e_{8}\right) \text {, } \\
& \kappa_{8^{\star}}^{-}\left(e_{3} e_{5}\right)=E_{1,7}+E_{2,8}+E_{3,5}+E_{4,6}=\frac{1}{2} \lambda_{8^{\star}}\left(e_{1} e_{7}+e_{2} e_{8}+e_{3} e_{5}+e_{4} e_{6}\right) \text {, } \\
& \kappa_{8^{\star}}^{-}\left(e_{3} e_{6}\right)=-E_{1,8}+E_{2,7}+E_{3,6}-E_{4,5}=\frac{1}{2} \lambda_{8^{\star}}\left(-e_{1} e_{8}+e_{2} e_{7}+e_{3} e_{6}-e_{4} e_{5}\right) \text {, } \\
& \kappa_{8^{\star}}^{-}\left(e_{3} e_{7}\right)=-E_{1,5}-E_{2,6}+E_{3,7}+E_{4,8}=\frac{1}{2} \lambda_{8^{\star}}\left(-e_{1} e_{5}-e_{2} e_{6}+e_{3} e_{7}+e_{4} e_{8}\right), \\
& \kappa_{8^{\star}}^{-}\left(e_{3} e_{8}\right)=E_{1,6}-E_{2,5}+E_{3,8}-E_{4,7}=\frac{1}{2} \lambda_{8^{\star}}\left(e_{1} e_{6}-e_{2} e_{5}+e_{3} e_{8}-e_{4} e_{7}\right) \text {, } \\
& \kappa_{8^{\star}}^{-}\left(e_{4} e_{5}\right)=-E_{1,8}+E_{2,7}-E_{3,6}+E_{4,5}=\frac{1}{2} \lambda_{8^{\star}}\left(-e_{1} e_{8}+e_{2} e_{7}-e_{3} e_{6}+e_{4} e_{5}\right) \text {, } \\
& \kappa_{8^{\star}}^{-}\left(e_{4} e_{6}\right)=-E_{1,7}-E_{2,8}+E_{3,5}+E_{4,6}=\frac{1}{2} \lambda_{8^{\star}}\left(-e_{1} e_{7}-e_{2} e_{8}+e_{3} e_{5}+e_{4} e_{6}\right) \text {, } \\
& \kappa_{8^{\star}}^{-}\left(e_{4} e_{7}\right)=E_{1,6}-E_{2,5}-E_{3,8}+E_{4,7}=\frac{1}{2} \lambda_{8^{\star}}\left(e_{1} e_{6}-e_{2} e_{5}-e_{3} e_{8}+e_{4} e_{7}\right) \text {, }
\end{aligned}
$$


174

Gerardo Arizmendi and Rafael Herrera

DE GRUYTER

$$
\begin{aligned}
& \kappa_{8^{\star}}^{-}\left(e_{4} e_{8}\right)=E_{1,5}+E_{2,6}+E_{3,7}+E_{4,8}=\frac{1}{2} \lambda_{8^{\star}}\left(e_{1} e_{5}+e_{2} e_{6}+e_{3} e_{7}+e_{4} e_{8}\right), \\
& \kappa_{8^{\star}}^{-}\left(e_{5} e_{6}\right)=E_{1,2}-E_{3,4}+E_{5,6}-E_{7,8}=\frac{1}{2} \lambda_{8^{\star}}\left(e_{1} e_{2}-e_{3} e_{4}+e_{5} e_{6}-e_{7} e_{8}\right), \\
& \kappa_{8^{\star}}^{-}\left(e_{5} e_{7}\right)=E_{1,3}+E_{2,4}+E_{5,7}+E_{6,8}=\frac{1}{2} \lambda_{8^{\star}}\left(e_{1} e_{3}+e_{2} e_{4}+e_{5} e_{7}+e_{6} e_{8}\right), \\
& \kappa_{8^{\star}}^{-}\left(e_{5} e_{8}\right)=-E_{1,4}+E_{2,3}+E_{5,8}-E_{6,7}=\frac{1}{2} \lambda_{8^{\star}}\left(-e_{1} e_{4}+e_{2} e_{3}+e_{5} e_{8}-e_{6} e_{7}\right), \\
& \kappa_{8^{\star}}^{-}\left(e_{6} e_{7}\right)=-E_{1,4}+E_{2,3}-E_{5,8}+E_{6,7}=\frac{1}{2} \lambda_{8^{\star}}\left(-e_{1} e_{4}+e_{2} e_{3}-e_{5} e_{8}+e_{6} e_{7}\right), \\
& \kappa_{8^{\star}}^{-}\left(e_{6} e_{8}\right)=-E_{1,3}-E_{2,4}+E_{5,7}+E_{6,8}=\frac{1}{2} \lambda_{8^{\star}}\left(-e_{1} e_{3}-e_{2} e_{4}+e_{5} e_{7}+e_{6} e_{8}\right), \\
& \kappa_{8^{\star}}^{-}\left(e_{7} e_{8}\right)=E_{1,2}-E_{3,4}-E_{5,6}+E_{7,8}=\frac{1}{2} \lambda_{8^{\star}}\left(e_{1} e_{2}-e_{3} e_{4}-e_{5} e_{6}+e_{7} e_{8}\right) .
\end{aligned}
$$

This means, in terms of the first diagram in (8),

$$
\begin{aligned}
& \sigma_{\star}\left(e_{1} e_{2}\right)=\frac{1}{2}\left(-e_{1} e_{2}-e_{3} e_{4}-e_{5} e_{6}-e_{7} e_{8}\right), \\
& \sigma_{\star}\left(e_{1} e_{3}\right)=\frac{1}{2}\left(-e_{1} e_{3}+e_{2} e_{4}-e_{5} e_{7}+e_{6} e_{8}\right) \text {, } \\
& \sigma_{\star}\left(e_{1} e_{4}\right)=\frac{1}{2}\left(-e_{1} e_{4}-e_{2} e_{3}+e_{5} e_{8}+e_{6} e_{7}\right) \text {, } \\
& \sigma_{\star}\left(e_{1} e_{5}\right)=\frac{1}{2}\left(-e_{1} e_{5}+e_{2} e_{6}+e_{3} e_{7}-e_{4} e_{8}\right), \\
& \sigma_{\star}\left(e_{1} e_{6}\right)=\frac{1}{2}\left(-e_{1} e_{6}-e_{2} e_{5}-e_{3} e_{8}-e_{4} e_{7}\right) \text {, } \\
& \sigma_{\star}\left(e_{1} e_{7}\right)=\frac{1}{2}\left(-e_{1} e_{7}+e_{2} e_{8}-e_{3} e_{5}+e_{4} e_{6}\right) \text {, } \\
& \sigma_{\star}\left(e_{1} e_{8}\right)=\frac{1}{2}\left(-e_{1} e_{8}-e_{2} e_{7}+e_{3} e_{6}+e_{4} e_{5}\right) \text {, } \\
& \sigma_{\star}\left(e_{2} e_{3}\right)=\frac{1}{2}\left(e_{1} e_{4}+e_{2} e_{3}+e_{5} e_{8}+e_{6} e_{7}\right) \text {, } \\
& \sigma_{\star}\left(e_{2} e_{4}\right)=\frac{1}{2}\left(-e_{1} e_{3}+e_{2} e_{4}+e_{5} e_{7}-e_{6} e_{8}\right) \text {, } \\
& \sigma_{\star}\left(e_{2} e_{5}\right)=\frac{1}{2}\left(e_{1} e_{6}+e_{2} e_{5}-e_{3} e_{8}-e_{4} e_{7}\right) \text {, } \\
& \sigma_{\star}\left(e_{2} e_{6}\right)=\frac{1}{2}\left(-e_{1} e_{5}+e_{2} e_{6}-e_{3} e_{7}+e_{4} e_{8}\right) \text {, } \\
& \sigma_{\star}\left(e_{2} e_{7}\right)=\frac{1}{2}\left(e_{1} e_{8}+e_{2} e_{7}+e_{3} e_{6}+e_{4} e_{5}\right) \text {, } \\
& \sigma_{\star}\left(e_{2} e_{8}\right)=\frac{1}{2}\left(-e_{1} e_{7}+e_{2} e_{8}+e_{3} e_{5}-e_{4} e_{6}\right) \text {, } \\
& \sigma_{\star}\left(e_{3} e_{4}\right)=\frac{1}{2}\left(e_{1} e_{2}+e_{3} e_{4}-e_{5} e_{6}-e_{7} e_{8}\right) \text {, } \\
& \sigma_{\star}\left(e_{3} e_{5}\right)=\frac{1}{2}\left(e_{1} e_{7}+e_{2} e_{8}+e_{3} e_{5}+e_{4} e_{6}\right) \text {, } \\
& \sigma_{\star}\left(e_{3} e_{6}\right)=\frac{1}{2}\left(-e_{1} e_{8}+e_{2} e_{7}+e_{3} e_{6}-e_{4} e_{5}\right) \text {, } \\
& \sigma_{\star}\left(e_{3} e_{7}\right)=\frac{1}{2}\left(-e_{1} e_{5}-e_{2} e_{6}+e_{3} e_{7}+e_{4} e_{8}\right) \text {, } \\
& \sigma_{\star}\left(e_{3} e_{8}\right)=\frac{1}{2}\left(e_{1} e_{6}-e_{2} e_{5}+e_{3} e_{8}-e_{4} e_{7}\right) \text {, } \\
& \sigma_{\star}\left(e_{4} e_{5}\right)=\frac{1}{2}\left(-e_{1} e_{8}+e_{2} e_{7}-e_{3} e_{6}+e_{4} e_{5}\right) \text {, } \\
& \sigma_{\star}\left(e_{4} e_{6}\right)=\frac{1}{2}\left(-e_{1} e_{7}-e_{2} e_{8}+e_{3} e_{5}+e_{4} e_{6}\right) \text {, } \\
& \sigma_{\star}\left(e_{4} e_{7}\right)=\frac{1}{2}\left(e_{1} e_{6}-e_{2} e_{5}-e_{3} e_{8}+e_{4} e_{7}\right), \\
& \sigma_{\star}\left(e_{4} e_{8}\right)=\frac{1}{2}\left(e_{1} e_{5}+e_{2} e_{6}+e_{3} e_{7}+e_{4} e_{8}\right) \text {, }
\end{aligned}
$$




$$
\begin{aligned}
& \sigma_{\star}\left(e_{5} e_{6}\right)=\frac{1}{2}\left(e_{1} e_{2}-e_{3} e_{4}+e_{5} e_{6}-e_{7} e_{8}\right), \\
& \sigma_{\star}\left(e_{5} e_{7}\right)=\frac{1}{2}\left(e_{1} e_{3}+e_{2} e_{4}+e_{5} e_{7}+e_{6} e_{8}\right), \\
& \sigma_{\star}\left(e_{5} e_{8}\right)=\frac{1}{2}\left(-e_{1} e_{4}+e_{2} e_{3}+e_{5} e_{8}-e_{6} e_{7}\right), \\
& \sigma_{\star}\left(e_{6} e_{7}\right)=\frac{1}{2}\left(-e_{1} e_{4}+e_{2} e_{3}-e_{5} e_{8}+e_{6} e_{7}\right), \\
& \sigma_{\star}\left(e_{6} e_{8}\right)=\frac{1}{2}\left(-e_{1} e_{3}-e_{2} e_{4}+e_{5} e_{7}+e_{6} e_{8}\right), \\
& \sigma_{\star}\left(e_{7} e_{8}\right)=\frac{1}{2}\left(e_{1} e_{2}-e_{3} e_{4}-e_{5} e_{6}+e_{7} e_{8}\right) .
\end{aligned}
$$

In other words, we have defined $\sigma \star$ in such a way that

$$
\lambda_{8^{\star}} \circ \sigma_{\star}=\kappa_{8^{\star}}^{-} .
$$

In order to show that $\sigma \star$ is of order 3, let us consider, for instance

$$
\sigma_{\star}\left(e_{1} e_{2}\right)=\frac{1}{2}\left(-e_{1} e_{2}-e_{3} e_{4}-e_{5} e_{6}-e_{7} e_{8}\right)
$$

\begin{tabular}{|c|c|c|c|c|c|c|c|c|c|c|c|c|c|c|c|c|c|c|c|c|c|c|c|c|c|c|c|c|}
\hline & -1 & 0 & 0 & 0 & 0 & 0 & 0 & 0 & 0 & 0 & 0 & 0 & 0 & 1 & 0 & 0 & 0 & 0 & 0 & 0 & 0 & 0 & 1 & 0 & 0 & 0 & 0 & 1 \\
\hline & 0 & -1 & 0 & 0 & 0 & 0 & 0 & 0 & -1 & 0 & 0 & 0 & 0 & 0 & 0 & 0 & 0 & 0 & 0 & 0 & 0 & 0 & 0 & 1 & 0 & 0 & -1 & 0 \\
\hline & 0 & 0 & -1 & 0 & 0 & 0 & 0 & 1 & 0 & 0 & 0 & 0 & 0 & 0 & 0 & 0 & 0 & 0 & 0 & 0 & 0 & 0 & 0 & 0 & -1 & -1 & 0 & 0 \\
\hline & 0 & 0 & 0 & -1 & 0 & 0 & 0 & 0 & 0 & 0 & -1 & 0 & 0 & 0 & 0 & 0 & -1 & 0 & 0 & 0 & 0 & 1 & 0 & 0 & 0 & 0 & 0 & 0 \\
\hline & 0 & 0 & 0 & 0 & -1 & 0 & 0 & 0 & 0 & 1 & 0 & 0 & 0 & 0 & 0 & 0 & 0 & 1 & 0 & 0 & 1 & 0 & 0 & 0 & 0 & 0 & 0 & 0 \\
\hline & 0 & 0 & 0 & 0 & 0 & -1 & 0 & 0 & 0 & 0 & 0 & 0 & -1 & 0 & 1 & 0 & 0 & 0 & 0 & -1 & 0 & 0 & 0 & 0 & 0 & 0 & 0 & 0 \\
\hline & 0 & 0 & 0 & 0 & 0 & 0 & -1 & 0 & 0 & 0 & 0 & 1 & 0 & 0 & 0 & -1 & 0 & 0 & -1 & 0 & 0 & 0 & 0 & 0 & 0 & 0 & 0 & 0 \\
\hline & 0 & 0 & -1 & 0 & 0 & 0 & 0 & 1 & 0 & 0 & 0 & 0 & 0 & 0 & 0 & 0 & 0 & 0 & 0 & 0 & 0 & 0 & 0 & 0 & 1 & 1 & 0 & 0 \\
\hline & 0 & 1 & 0 & 0 & 0 & 0 & 0 & 0 & 1 & 0 & 0 & 0 & 0 & 0 & 0 & 0 & 0 & 0 & 0 & 0 & 0 & 0 & 0 & 1 & 0 & 0 & -1 & 0 \\
\hline & 0 & 0 & 0 & 0 & -1 & 0 & 0 & 0 & 0 & 1 & 0 & 0 & 0 & 0 & 0 & 0 & 0 & -1 & 0 & 0 & -1 & 0 & 0 & 0 & 0 & 0 & 0 & 0 \\
\hline & 0 & 0 & 0 & 1 & 0 & 0 & 0 & 0 & 0 & 0 & 1 & 0 & 0 & 0 & 0 & 0 & -1 & 0 & 0 & 0 & 0 & 1 & 0 & 0 & 0 & 0 & 0 & 0 \\
\hline & 0 & 0 & 0 & 0 & 0 & 0 & -1 & 0 & 0 & 0 & 0 & 1 & 0 & 0 & 0 & 1 & 0 & 0 & 1 & 0 & 0 & 0 & 0 & 0 & 0 & 0 & 0 & 0 \\
\hline & 0 & 0 & 0 & 0 & 0 & 1 & 0 & 0 & 0 & 0 & 0 & 0 & 1 & 0 & 1 & 0 & 0 & 0 & 0 & -1 & 0 & 0 & 0 & 0 & 0 & 0 & 0 & 0 \\
\hline$=1$ & -1 & 0 & 0 & 0 & 0 & 0 & 0 & 0 & 0 & 0 & 0 & 0 & 0 & 1 & 0 & 0 & 0 & 0 & 0 & 0 & 0 & 0 & -1 & 0 & 0 & 0 & 0 & -1 \\
\hline$=-$ & 0 & 0 & 0 & 0 & 0 & -1 & 0 & 0 & 0 & 0 & 0 & 0 & 1 & 0 & 1 & 0 & 0 & 0 & 0 & 1 & 0 & 0 & 0 & 0 & 0 & 0 & 0 & 0 \\
\hline & 0 & 0 & 0 & 0 & 0 & 0 & 1 & 0 & 0 & 0 & 0 & 1 & 0 & 0 & 0 & 1 & 0 & 0 & -1 & 0 & 0 & 0 & 0 & 0 & 0 & 0 & 0 & 0 \\
\hline & 0 & 0 & 0 & 1 & 0 & 0 & 0 & 0 & 0 & 0 & -1 & 0 & 0 & 0 & 0 & 0 & 1 & 0 & 0 & 0 & 0 & 1 & 0 & 0 & 0 & 0 & 0 & 0 \\
\hline & 0 & 0 & 0 & 0 & -1 & 0 & 0 & 0 & 0 & -1 & 0 & 0 & 0 & 0 & 0 & 0 & 0 & 1 & 0 & 0 & -1 & 0 & 0 & 0 & 0 & 0 & 0 & 0 \\
\hline & 0 & 0 & 0 & 0 & 0 & 0 & 1 & 0 & 0 & 0 & 0 & 1 & 0 & 0 & 0 & -1 & 0 & 0 & 1 & 0 & 0 & 0 & 0 & 0 & 0 & 0 & 0 & 0 \\
\hline & 0 & 0 & 0 & 0 & 0 & 1 & 0 & 0 & 0 & 0 & 0 & 0 & -1 & 0 & 1 & 0 & 0 & 0 & 0 & 1 & 0 & 0 & 0 & 0 & 0 & 0 & 0 & 0 \\
\hline & 0 & 0 & 0 & 0 & -1 & 0 & 0 & 0 & 0 & -1 & 0 & 0 & 0 & 0 & 0 & 0 & 0 & -1 & 0 & 0 & 1 & 0 & 0 & 0 & 0 & 0 & 0 & 0 \\
\hline & 0 & 0 & 0 & -1 & 0 & 0 & 0 & 0 & 0 & 0 & 1 & 0 & 0 & 0 & 0 & 0 & 1 & 0 & 0 & 0 & 0 & 1 & 0 & 0 & 0 & 0 & 0 & 0 \\
\hline & -1 & 0 & 0 & 0 & 0 & 0 & 0 & 0 & 0 & 0 & 0 & 0 & 0 & -1 & 0 & 0 & 0 & 0 & 0 & 0 & 0 & 0 & 1 & 0 & 0 & 0 & 0 & -1 \\
\hline & 0 & -1 & 0 & 0 & 0 & 0 & 0 & 0 & 1 & 0 & 0 & 0 & 0 & 0 & 0 & 0 & 0 & 0 & 0 & 0 & 0 & 0 & 0 & 1 & 0 & 0 & 1 & 0 \\
\hline & 0 & 0 & 1 & 0 & 0 & 0 & 0 & 1 & 0 & 0 & 0 & 0 & 0 & 0 & 0 & 0 & 0 & 0 & 0 & 0 & 0 & 0 & 0 & 0 & 1 & -1 & 0 & 0 \\
\hline & 0 & 0 & 1 & 0 & 0 & 0 & 0 & 1 & 0 & 0 & 0 & 0 & 0 & 0 & 0 & 0 & 0 & 0 & 0 & 0 & 0 & 0 & 0 & 0 & -1 & 1 & 0 & 0 \\
\hline & 0 & 1 & 0 & 0 & 0 & 0 & 0 & 0 & -1 & 0 & 0 & 0 & 0 & 0 & 0 & 0 & 0 & 0 & 0 & 0 & 0 & 0 & 0 & 1 & 0 & 0 & 1 & 0 \\
\hline & -1 & 0 & 0 & 0 & 0 & 0 & 0 & 0 & 0 & 0 & 0 & 0 & 0 & -1 & 0 & 0 & 0 & 0 & 0 & 0 & 0 & 0 & -1 & 0 & 0 & 0 & 0 & 1 \\
\hline
\end{tabular}

Then

$$
\begin{aligned}
\sigma_{\star}\left(\sigma_{\star}\left(e_{1} e_{2}\right)\right)= & \frac{1}{2}\left(-\sigma_{\star}\left(e_{1} e_{2}\right)-\sigma_{\star}\left(e_{3} e_{4}\right)-\sigma_{\star}\left(e_{5} e_{6}\right)-\sigma_{\star}\left(e_{7} e_{8}\right)\right) \\
= & \frac{1}{4}\left(-\left(-e_{1} e_{2}-e_{3} e_{4}-e_{5} e_{6}-e_{7} e_{8}\right)-\left(e_{1} e_{2}+e_{3} e_{4}-e_{5} e_{6}-e_{7} e_{8}\right)\right. \\
& \left.-\left(e_{1} e_{2}-e_{3} e_{4}+e_{5} e_{6}-e_{7} e_{8}\right)-\left(e_{1} e_{2}-e_{3} e_{4}-e_{5} e_{6}+e_{7} e_{8}\right)\right) \\
= & \frac{1}{2}\left(-e_{1} e_{2}+e_{3} e_{4}+e_{5} e_{6}+e_{7} e_{8}\right),
\end{aligned}
$$

and

$$
\begin{aligned}
\sigma_{\star}\left(\sigma_{\star}\left(\sigma_{\star}\left(e_{1} e_{2}\right)\right)\right)= & \frac{1}{2}\left(-\sigma_{\star}\left(e_{1} e_{2}\right)+\sigma_{\star}\left(e_{3} e_{4}\right)+\sigma_{\star}\left(e_{5} e_{6}\right)+\sigma_{\star}\left(e_{7} e_{8}\right)\right) \\
= & \frac{1}{4}\left(-\left(-e_{1} e_{2}-e_{3} e_{4}-e_{5} e_{6}-e_{7} e_{8}\right)+\left(e_{1} e_{2}+e_{3} e_{4}-e_{5} e_{6}-e_{7} e_{8}\right)\right. \\
& \left.+\left(e_{1} e_{2}-e_{3} e_{4}+e_{5} e_{6}-e_{7} e_{8}\right)+\left(e_{1} e_{2}-e_{3} e_{4}-e_{5} e_{6}+e_{7} e_{8}\right)\right) \\
= & e_{1} e_{2} .
\end{aligned}
$$

All the other cases are similar. In fact, using the standard ordered basis $\left\{e_{1} e_{2}, e_{1} e_{3}, \ldots, e_{7} e_{8}\right\}$ of $\mathfrak{s p i n}(8)$ we have the matrix representation

which one can verify is of order 3. Furthermore, the map $\sigma_{\star}$ has eigenvalues

$$
e^{\frac{2 \pi i}{3}}, e^{\frac{-2 \pi i}{3}}, 1,
$$


with multiplicities 7,7 and 14 respectively. The eigenspace corresponding to 1 is generated by

$$
\begin{aligned}
& \left\{e_{2} e_{3}+e_{6} e_{7},-e_{2} e_{4}+e_{6} e_{8},-e_{3} e_{4}+e_{7} e_{8},-e_{2} e_{6}+e_{3} e_{7},-e_{2} e_{5}+e_{3} e_{8}, e_{2} e_{7}+e_{4} e_{5},-e_{2} e_{8}+e_{4} e_{6},\right. \\
& \left.-e_{2} e_{5}+e_{4} e_{7}, e_{2} e_{6}+e_{4} e_{8},-e_{3} e_{4}+e_{5} e_{6}, e_{2} e_{4}+e_{5} e_{7}, e_{2} e_{3}+e_{5} e_{8}, e_{2} e_{8}+e_{3} e_{5}, e_{2} e_{7}+e_{3} e_{6},\right\}
\end{aligned}
$$

which generates a copy of $\mathfrak{g}_{2} \subset \mathfrak{s p i n}(8) \subset C l_{8}^{0}$ (see $[4,13,17]$ for definitions of $\mathfrak{g}_{2}$ and $G_{2}$ ). Note that none of the generators includes the vector $e_{1}$, which makes this copy of $\mathfrak{g}_{2}$ a subalgebra of the copy of $\mathfrak{s p i n}(7)$ generated by the span of $\left\{e_{2}, e_{3}, e_{4}, e_{5}, e_{6}, e_{7}, e_{8}\right\}$. This copy of $\mathfrak{g}_{2}$ annihilates the basic positive spinor

$$
\frac{1}{\sqrt{2}}\left(u_{0}-u_{15}\right) \in \tilde{\Delta}_{8}^{+}
$$

so that $\tilde{\Delta}_{8}^{+}=\mathbf{1} \oplus \mathbb{R}^{7}$ under $\mathfrak{g}_{2}$ and also annihilates the basic negative spinor

$$
\frac{i}{\sqrt{2}}\left(u_{1}-u_{14}\right) \in \tilde{\Delta}_{8}^{-}
$$

under Clifford multiplication, so that $\tilde{\Delta}_{8}^{-}=\mathbf{1} \oplus \mathbb{R}^{7}$ under $\mathfrak{g}_{2}$. The matrix representation for a general element

$$
\begin{aligned}
& \alpha_{1}\left(e_{2} e_{3}+e_{6} e_{7}\right)+\alpha_{2}\left(-e_{2} e_{4}+e_{6} e_{8}\right)+\alpha_{3}\left(-e_{3} e_{4}+e_{7} e_{8}\right)+\alpha_{4}\left(-e_{2} e_{6}+e_{3} e_{7}\right)+\alpha_{5}\left(-e_{2} e_{5}+e_{3} e_{8}\right) \\
& +\alpha_{6}\left(e_{2} e_{7}+e_{4} e_{5}\right)+\alpha_{7}\left(-e_{2} e_{8}+e_{4} e_{6}\right)+\alpha_{8}\left(-e_{2} e_{5}+e_{4} e_{7}\right)+\alpha_{9}\left(e_{2} e_{6}+e_{4} e_{8}\right)+\alpha_{10}\left(-e_{3} e_{4}+e_{5} e_{6}\right) \\
& +\alpha_{11}\left(e_{2} e_{4}+e_{5} e_{7}\right)+\alpha_{12}\left(e_{2} e_{3}+e_{5} e_{8}\right)+\alpha_{13}\left(e_{2} e_{8}+e_{3} e_{5}\right)+\alpha_{14}\left(e_{2} e_{7}+e_{3} e_{6}\right)
\end{aligned}
$$

on both $\tilde{\Delta}_{8}^{+}$and $\tilde{\Delta}_{8}^{-}$, is

$$
2\left(\begin{array}{cccccccc}
0 & 0 & 0 & 0 & 0 & 0 & 0 & 0 \\
0 & 0 & \alpha_{1}+\alpha_{12} & -\alpha_{2}+\alpha_{11} & -\alpha_{5}-\alpha_{8} & -\alpha_{4}+\alpha_{9} & \alpha_{6}+\alpha_{14} & -\alpha_{7}+\alpha_{13} \\
0 & -\alpha_{1}-\alpha_{12} & 0 & -\alpha_{3}-\alpha_{10} & \alpha_{13} & \alpha_{14} & \alpha_{4} & \alpha_{5} \\
0 & \alpha_{2}-\alpha_{11} & \alpha_{3}+\alpha_{10} & 0 & \alpha_{6} & \alpha_{7} & \alpha_{8} & \alpha_{9} \\
0 & \alpha_{5}+\alpha_{8} & -\alpha_{13} & -\alpha_{6} & 0 & \alpha_{10} & \alpha_{11} & \alpha_{12} \\
0 & \alpha_{4}-\alpha_{9} & -\alpha_{14} & -\alpha_{7} & -\alpha_{10} & 0 & \alpha_{1} & \alpha_{2} \\
0 & -\alpha_{6}-\alpha_{14} & -\alpha_{4} & -\alpha_{8} & -\alpha_{11} & -\alpha_{1} & 0 & \alpha_{3} \\
0 & \alpha_{7}-\alpha_{13} & -\alpha_{5} & -\alpha_{9} & -\alpha_{12} & -\alpha_{2} & -\alpha_{3} & 0
\end{array}\right) .
$$

Let us compute an explicit element of the group $G_{2}$. Consider the element $e_{2} e_{3}+e_{6} e_{7} \in \mathfrak{g}_{2} \subset \mathfrak{s p i n}(8) \subset C l_{8}^{0}$, and the one parameter subgroup

$$
\begin{aligned}
\exp \left(t\left(e_{2} e_{3}+e_{6} e_{7}\right)\right) & =\exp \left(t e_{2} e_{3}\right) \exp \left(t e_{6} e_{7}\right) \\
& =\left(\cos (t)+\sin (t) e_{2} e_{3}\right)\left(\cos (t)+\sin (t) e_{6} e_{7}\right) \\
& =\frac{1}{2}\left(-e_{2} e_{3} e_{6} e_{7} \cos (2 t)+e_{2} e_{3} e_{6} e_{7}+e_{2} e_{3} \sin (2 t)+e_{6} e_{7} \sin (2 t)+\cos (2 t)+1\right) \\
& =\frac{1}{2}\left(e_{2} e_{3} e_{6} e_{7}(1-\cos (2 t))+\left(e_{2} e_{3}+e_{6} e_{7}\right) \sin (2 t)+\cos (2 t)+1\right) \\
& \in G_{2} \subset \operatorname{Spin}(7) \subset \operatorname{Spin}(8) \subset C l_{8}^{0}
\end{aligned}
$$

Its image under $\kappa_{8}^{-}$is

$$
\begin{aligned}
& \kappa_{8}^{-}\left(\exp \left(t\left(e_{2} e_{3}+e_{6} e_{7}\right)\right)\right)=\left(\cos (t) \operatorname{Id}+\sin (t) \kappa_{8}^{-}\left(e_{2} e_{3}\right)\right)\left(\cos (t) \operatorname{Id}+\sin (t) \kappa_{8}^{-}\left(e_{6} e_{7}\right)\right) \\
&=\left(\begin{array}{cccccccc}
1 & 0 & 0 & 0 & 0 & 0 & 0 & 0 \\
0 & \cos (2 t) & -\sin (2 t) & 0 & 0 & 0 & 0 & 0 \\
0 & \sin (2 t) & \cos (2 t) & 0 & 0 & 0 & 0 & 0 \\
0 & 0 & 0 & 1 & 0 & 0 & 0 & 0 \\
0 & 0 & 0 & 0 & 1 & 0 & 0 & 0 \\
0 & 0 & 0 & 0 & 0 & \cos (2 t) & -\sin (2 t) & 0 \\
0 & 0 & 0 & 0 & 0 & \sin (2 t) & \cos (2 t) & 0 \\
0 & 0 & 0 & 0 & 0 & 0 & 0 & 1
\end{array}\right)
\end{aligned}
$$


$\in \quad \kappa_{8}^{-}\left(G_{2}\right) \subset S O(7) \subset S O(8)$.

The eigenspace corresponding to $e^{\frac{2 \pi i}{3}}$ is generated by

$$
\begin{aligned}
& \left\{e_{6} e_{8}-e_{5} e_{7}+e_{2} e_{4}+i e_{1} e_{3} \sqrt{3}, e_{4} e_{7}+e_{3} e_{8}+e_{2} e_{5}-i e_{1} e_{6} \sqrt{3}, e_{4} e_{6}-e_{3} e_{5}+e_{2} e_{8}+i e_{1} e_{7} \sqrt{3},\right. \\
& e_{7} e_{8}+e_{5} e_{6}+e_{3} e_{4}-i e_{1} e_{2} \sqrt{3}, e_{4} e_{5}+e_{3} e_{6}-e_{2} e_{7}+i e_{1} e_{8} \sqrt{3}, e_{4} e_{8}-e_{3} e_{7}-e_{2} e_{6}-i e_{1} e_{5} \sqrt{3}, \\
& \left.e_{6} e_{7}+e_{5} e_{8}-e_{2} e_{3}+i e_{1} e_{4} \sqrt{3}\right\}
\end{aligned}
$$

and eigenspace corresponding to $e^{\frac{-2 \pi i}{3}}$ is generated by

$$
\begin{aligned}
& \left\{e_{6} e_{8}-e_{5} e_{7}+e_{2} e_{4}-i e_{1} e_{3} \sqrt{3}, e_{4} e_{7}+e_{3} e_{8}+e_{2} e_{5}+i e_{1} e_{6} \sqrt{3}, e_{4} e_{6}-e_{3} e_{5}+e_{2} e_{8}-i e_{1} e_{7} \sqrt{3},\right. \\
& e_{7} e_{8}+e_{5} e_{6}+e_{3} e_{4}+i e_{1} e_{2} \sqrt{3}, e_{4} e_{5}+e_{3} e_{6}-e_{2} e_{7}-i e_{1} e_{8} \sqrt{3}, e_{4} e_{8}-e_{3} e_{7}-e_{2} e_{6}+i e_{1} e_{5} \sqrt{3}, \\
& \left.e_{6} e_{7}+e_{5} e_{8}-e_{2} e_{3}-i e_{1} e_{4} \sqrt{3}\right\}
\end{aligned}
$$

\subsubsection{The endomorphism $\tau$ *}

Using the ordered basis of spinors, one can compute the endomorphisms corresponding to the elements $e_{i} e_{j} \in \mathfrak{s p i n}(8), 1 \leq i<j \leq 8$, under the map $\kappa_{8^{\star}}^{+}$and, in turn, express those endomorphisms as images of elements of $\mathfrak{s p i n}(8)$ under $\lambda_{8^{*}}$ :

$$
\begin{aligned}
& \kappa_{8^{\star}}^{+}\left(e_{1} e_{2}\right)=E_{1,2}+E_{3,4}+E_{5,6}+E_{7,8}=\frac{1}{2} \lambda_{8^{\star}}\left(e_{1,2}+e_{3,4}+e_{5,6}+e_{7,8}\right), \\
& \kappa_{8^{\star}}^{+}\left(e_{1} e_{3}\right)=E_{1,3}-E_{2,4}+E_{5,7}-E_{6,8}=\frac{1}{2} \lambda_{8^{\star}}\left(e_{1,3}-e_{2,4}+e_{5,7}-e_{6,8}\right), \\
& \kappa_{8^{\star}}^{+}\left(e_{1} e_{4}\right)=E_{1,4}+E_{2,3}-E_{5,8}-E_{6,7}=\frac{1}{2} \lambda_{8^{\star}}\left(e_{1,4}+e_{2,3}-e_{5,8}-e_{6,7}\right), \\
& \kappa_{8^{\star}}^{+}\left(e_{1} e_{5}\right)=E_{1,5}-E_{2,6}-E_{3,7}+E_{4,8}=\frac{1}{2} \lambda_{8^{\star}}\left(e_{1,5}-e_{2,6}-e_{3,7}+e_{4,8}\right), \\
& \kappa_{8^{\star}}^{+}\left(e_{1} e_{6}\right)=E_{1,6}+E_{2,5}+E_{3,8}+E_{4,7}=\frac{1}{2} \lambda_{8^{\star}}\left(e_{1,6}+e_{2,5}+e_{3,8}+e_{4,7}\right) \text {, } \\
& \kappa_{8^{\star}}^{+}\left(e_{1} e_{7}\right)=E_{1,7}-E_{2,8}+E_{3,5}-E_{4,6}=\frac{1}{2} \lambda_{8^{\star}}\left(e_{1,7}-e_{2,8}+e_{3,5}-e_{4,6}\right), \\
& \kappa_{8^{\star}}^{+}\left(e_{1} e_{8}\right)=E_{1,8}+E_{2,7}-E_{3,6}-E_{4,5}=\frac{1}{2} \lambda_{8^{\star}}\left(e_{1,8}+e_{2,7}-e_{3,6}-e_{4,5}\right), \\
& \kappa_{8^{\star}}^{+}\left(e_{2} e_{3}\right)=E_{1,4}+E_{2,3}+E_{5,8}+E_{6,7}=\frac{1}{2} \lambda_{8^{\star}}\left(e_{1,4}+e_{2,3}+e_{5,8}+e_{6,7}\right), \\
& \kappa_{8^{\star}}^{+}\left(e_{2} e_{4}\right)=-E_{1,3}+E_{2,4}+E_{5,7}-E_{6,8}=\frac{1}{2} \lambda_{8^{\star}}\left(-e_{1,3}+e_{2,4}+e_{5,7}-e_{6,8}\right) \text {, } \\
& \kappa_{8^{\star}}^{+}\left(e_{2} e_{5}\right)=E_{1,6}+E_{2,5}-E_{3,8}-E_{4,7}=\frac{1}{2} \lambda_{8^{\star}}\left(e_{1,6}+e_{2,5}-e_{3,8}-e_{4,7}\right), \\
& \kappa_{8^{\star}}^{+}\left(e_{2} e_{6}\right)=-E_{1,5}+E_{2,6}-E_{3,7}+E_{4,8}=\frac{1}{2} \lambda_{8^{\star}}\left(-e_{1,5}+e_{2,6}-e_{3,7}+e_{4,8}\right) \text {, } \\
& \kappa_{8^{\star}}^{+}\left(e_{2} e_{7}\right)=E_{1,8}+E_{2,7}+E_{3,6}+E_{4,5}=\frac{1}{2} \lambda_{8^{\star}}\left(e_{1,8}+e_{2,7}+e_{3,6}+e_{4,5}\right) \text {, } \\
& \kappa_{8^{\star}}^{+}\left(e_{2} e_{8}\right)=-E_{1,7}+E_{2,8}+E_{3,5}-E_{4,6}=\frac{1}{2} \lambda_{8^{\star}}\left(-e_{1,7}+e_{2,8}+e_{3,5}-e_{4,6}\right) \text {, } \\
& \kappa_{8^{\star}}^{+}\left(e_{3} e_{4}\right)=E_{1,2}+E_{3,4}-E_{5,6}-E_{7,8}=\frac{1}{2} \lambda_{8^{\star}}\left(e_{1,2}+e_{3,4}-e_{5,6}-e_{7,8}\right), \\
& \kappa_{8^{\star}}^{+}\left(e_{3} e_{5}\right)=E_{1,7}+E_{2,8}+E_{3,5}+E_{4,6}=\frac{1}{2} \lambda_{8^{\star}}\left(e_{1,7}+e_{2,8}+e_{3,5}+e_{4,6}\right), \\
& \kappa_{8^{\star}}^{+}\left(e_{3} e_{6}\right)=-E_{1,8}+E_{2,7}+E_{3,6}-E_{4,5}=\frac{1}{2} \lambda_{8^{\star}}\left(-e_{1,8}+e_{2,7}+e_{3,6}-e_{4,5}\right) \text {, } \\
& \kappa_{8^{\star}}^{+}\left(e_{3} e_{7}\right)=-E_{1,5}-E_{2,6}+E_{3,7}+E_{4,8}=\frac{1}{2} \lambda_{8^{\star}}\left(-e_{1,5}-e_{2,6}+e_{3,7}+e_{4,8}\right) \text {, } \\
& \kappa_{8^{\star}}^{+}\left(e_{3} e_{8}\right)=E_{1,6}-E_{2,5}+E_{3,8}-E_{4,7}=\frac{1}{2} \lambda_{8^{\star}}\left(e_{1,6}-e_{2,5}+e_{3,8}-e_{4,7}\right),
\end{aligned}
$$


178

Gerardo Arizmendi and Rafael Herrera

DE GRUYTER

$$
\begin{aligned}
& \kappa_{8^{\star}}^{+}\left(e_{4} e_{5}\right)=-E_{1,8}+E_{2,7}-E_{3,6}+E_{4,5}=\frac{1}{2} \lambda_{8^{\star}}\left(-e_{1,8}+e_{2,7}-e_{3,6}+e_{4,5}\right), \\
& \kappa_{8^{\star}}^{+}\left(e_{4} e_{6}\right)=-E_{1,7}-E_{2,8}+E_{3,5}+E_{4,6}=\frac{1}{2} \lambda_{8^{\star}}\left(-e_{1,7}-e_{2,8}+e_{3,5}+e_{4,6}\right), \\
& \kappa_{8^{\star}}^{+}\left(e_{4} e_{7}\right)=E_{1,6}-E_{2,5}-E_{3,8}+E_{4,7}=\frac{1}{2} \lambda_{8^{\star}}\left(e_{1,6}-e_{2,5}-e_{3,8}+e_{4,7}\right), \\
& \kappa_{8^{\star}}^{+}\left(e_{4} e_{8}\right)=E_{1,5}+E_{2,6}+E_{3,7}+E_{4,8}=\frac{1}{2} \lambda_{8^{\star}}\left(e_{1,5}+e_{2,6}+e_{3,7}+e_{4,8}\right), \\
& \kappa_{8^{\star}}^{+}\left(e_{5} e_{6}\right)=E_{1,2}-E_{3,4}+E_{5,6}-E_{7,8}=\frac{1}{2} \lambda_{8^{\star}}\left(e_{1,2}-e_{3,4}+e_{5,6}-e_{7,8}\right), \\
& \kappa_{8^{\star}}^{+}\left(e_{5} e_{7}\right)=E_{1,3}+E_{2,4}+E_{5,7}+E_{6,8}=\frac{1}{2} \lambda_{8^{\star}}\left(e_{1,3}+e_{2,4}+e_{5,7}+e_{6,8}\right), \\
& \kappa_{8^{\star}}^{+}\left(e_{5} e_{8}\right)=-E_{1,4}+E_{2,3}+E_{5,8}-E_{6,7}=\frac{1}{2} \lambda_{8^{\star}}\left(-e_{1,4}+e_{2,3}+e_{5,8}-e_{6,7}\right), \\
& \kappa_{8^{\star}}^{+}\left(e_{6} e_{7}\right)=-E_{1,4}+E_{2,3}-E_{5,8}+E_{6,7}=\frac{1}{2} \lambda_{8^{\star}}\left(-e_{1,4}+e_{2,3}-e_{5,8}+e_{6,7}\right), \\
& \kappa_{8^{\star}}^{+}\left(e_{6} e_{8}\right)=-E_{1,3}-E_{2,4}+E_{5,7}+E_{6,8}=\frac{1}{2} \lambda_{8^{\star}}\left(-e_{1,3}-e_{2,4}+e_{5,7}+e_{6,8}\right), \\
& \kappa_{8^{\star}}^{+}\left(e_{7} e_{8}\right)=E_{1,2}-E_{3,4}-E_{5,6}+E_{7,8}=\frac{1}{2} \lambda_{8^{\star}}\left(e_{1,2}-e_{3,4}-e_{5,6}+e_{7,8}\right),
\end{aligned}
$$

This means, in terms of the second diagram in (8),

$$
\begin{aligned}
\tau \star\left(e_{1} e_{2}\right) & =\frac{1}{2}\left(e_{1} e_{2}+e_{3} e_{4}+e_{5} e_{6}+e_{7} e_{8}\right), \\
\tau \star\left(e_{1} e_{3}\right) & =\frac{1}{2}\left(e_{1} e_{3}-e_{2} e_{4}+e_{5} e_{7}-e_{6} e_{8}\right), \\
\tau \star\left(e_{1} e_{4}\right) & =\frac{1}{2}\left(e_{1} e_{4}+e_{2} e_{3}-e_{5} e_{8}-e_{6} e_{7}\right), \\
\tau \star\left(e_{1} e_{5}\right) & =\frac{1}{2}\left(e_{1} e_{5}-e_{2} e_{6}-e_{3} e_{7}+e_{4} e_{8}\right), \\
\tau \star\left(e_{1} e_{6}\right) & =\frac{1}{2}\left(e_{1} e_{6}+e_{2} e_{5}+e_{3} e_{8}+e_{4} e_{7}\right), \\
\tau \star\left(e_{1} e_{7}\right) & =\frac{1}{2}\left(e_{1} e_{7}-e_{2} e_{8}+e_{3} e_{5}-e_{4} e_{6}\right), \\
\tau \star\left(e_{1} e_{8}\right) & =\frac{1}{2}\left(e_{1} e_{8}+e_{2} e_{7}-e_{3} e_{6}-e_{4} e_{5}\right), \\
\tau \star\left(e_{2} e_{3}\right) & =\frac{1}{2}\left(e_{1} e_{4}+e_{2} e_{3}+e_{5} e_{8}+e_{6} e_{7}\right), \\
\tau \star\left(e_{2} e_{4}\right) & =\frac{1}{2}\left(-e_{1} e_{3}+e_{2} e_{4}+e_{5} e_{7}-e_{6} e_{8}\right), \\
\tau \star\left(e_{2} e_{5}\right) & =\frac{1}{2}\left(e_{1} e_{6}+e_{2} e_{5}-e_{3} e_{8}-e_{4} e_{7}\right), \\
\tau \star\left(e_{2} e_{6}\right) & =\frac{1}{2}\left(-e_{1} e_{5}+e_{2} e_{6}-e_{3} e_{7}+e_{4} e_{8}\right), \\
\tau \star\left(e_{4} e_{5}\right) & =\frac{1}{2}\left(-e_{1} e_{8}+e_{2} e_{7}-e_{3} e_{6}+e_{4} e_{5}\right), \\
\tau \star\left(e_{2} e_{7}\right) & =\frac{1}{2}\left(e_{1} e_{8}+e_{2} e_{7}+e_{3} e_{6}+e_{4} e_{5}\right), \\
\tau \star\left(e_{2} e_{8}\right) & =\frac{1}{2}\left(-e_{1} e_{7}+e_{2} e_{8}+e_{3} e_{5}-e_{4} e_{6}\right), \\
\tau \star\left(e_{3} e_{4}\right) & =\frac{1}{2}\left(e_{1} e_{2}+e_{3} e_{4}-e_{5} e_{6}-e_{7} e_{8}\right), \\
\tau \star\left(e_{3} e_{6}\right) & =\frac{1}{2}\left(e_{1} e_{7}+e_{2} e_{8}+e_{3} e_{5}+e_{4} e_{6}\right), \\
\left.\tau \star e_{3} e_{8}\right) & =\frac{1}{2}\left(-e_{1} e_{5}-e_{2} e_{6}+e_{3} e_{7}+e_{4} e_{8}\right),
\end{aligned}
$$




$$
\begin{aligned}
\tau \star\left(e_{4} e_{6}\right) & =\frac{1}{2}\left(-e_{1} e_{7}-e_{2} e_{8}+e_{3} e_{5}+e_{4} e_{6}\right), \\
\tau \star\left(e_{4} e_{7}\right) & =\frac{1}{2}\left(e_{1} e_{6}-e_{2} e_{5}-e_{3} e_{8}+e_{4} e_{7}\right), \\
\tau \star\left(e_{4} e_{8}\right) & =\frac{1}{2}\left(e_{1} e_{5}+e_{2} e_{6}+e_{3} e_{7}+e_{4} e_{8}\right), \\
\tau \star\left(e_{5} e_{6}\right) & =\frac{1}{2}\left(e_{1} e_{2}-e_{3} e_{4}+e_{5} e_{6}-e_{7} e_{8}\right), \\
\tau \star\left(e_{5} e_{7}\right) & =\frac{1}{2}\left(e_{1} e_{3}+e_{2} e_{4}+e_{5} e_{7}+e_{6} e_{8}\right), \\
\tau \star\left(e_{5} e_{8}\right) & =\frac{1}{2}\left(-e_{1} e_{4}+e_{2} e_{3}+e_{5} e_{8}-e_{6} e_{7}\right), \\
\tau \star\left(e_{6} e_{7}\right) & =\frac{1}{2}\left(-e_{1} e_{4}+e_{2} e_{3}-e_{5} e_{8}+e_{6} e_{7}\right), \\
\tau \star\left(e_{6} e_{8}\right) & =\frac{1}{2}\left(-e_{1} e_{3}-e_{2} e_{4}+e_{5} e_{7}+e_{6} e_{8}\right), \\
\tau \star\left(e_{7} e_{8}\right) & =\frac{1}{2}\left(e_{1} e_{2}-e_{3} e_{4}-e_{5} e_{6}+e_{7} e_{8}\right) .
\end{aligned}
$$

In other words, we have defined $\tau \star$ in such a way that

$$
\lambda_{8^{\star}} \circ \tau_{\star}=\kappa_{8^{\star}}^{+} .
$$

As before, using the stardard ordered basis of $\mathfrak{s p i n}(8)$, we have the matrix representation

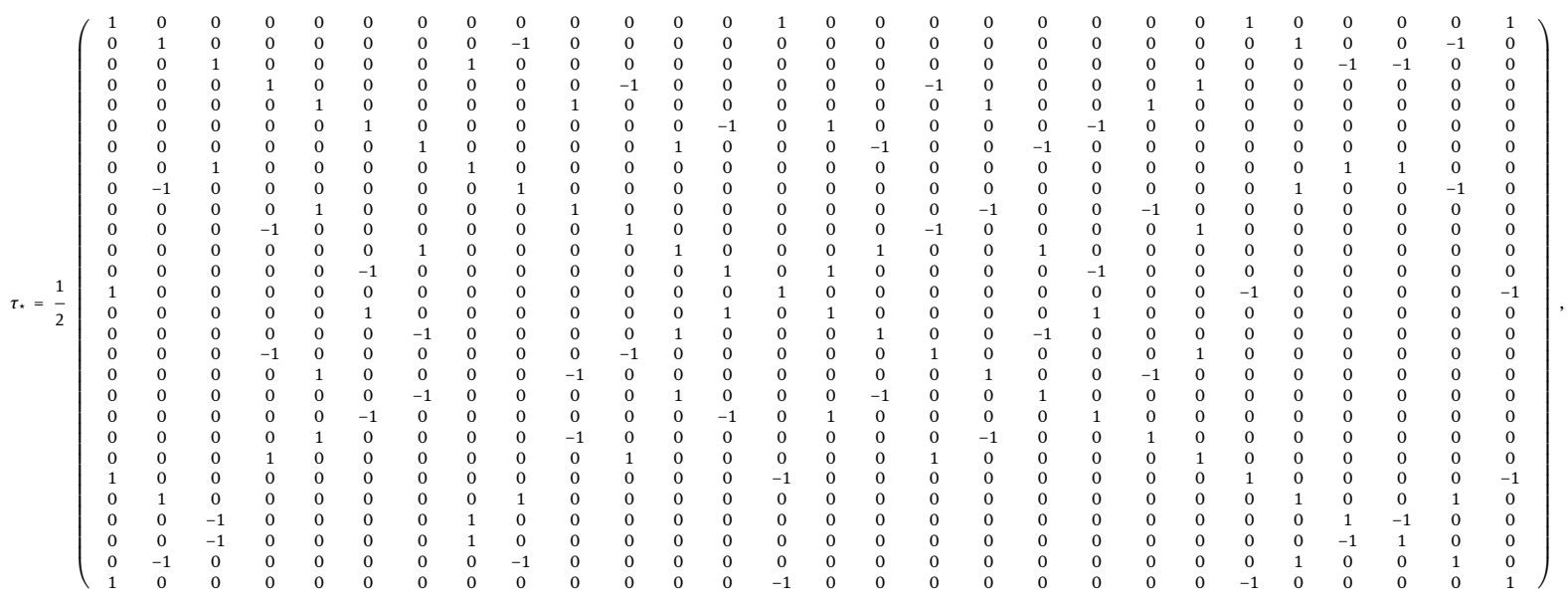

which one can verify is of order 2 . The map $\tau \star$ has eigenvalues

$$
1,-1
$$

with multiplicities 21 and 7 respectively. The eigenspace corresponding to 1 is generated by

$$
\begin{aligned}
& \left\{e_{1} e_{4}+e_{2} e_{3},-e_{1} e_{3}+e_{2} e_{4}, e_{1} e_{6}+e_{2} e_{5},-e_{1} e_{5}+e_{2} e_{6}, e_{1} e_{8}+e_{2} e_{7},-e_{1} e_{7}+e_{2} e_{8}, e_{1} e_{2}+e_{3} e_{4},\right. \\
& e_{1} e_{7}+e_{3} e_{5},-e_{1} e_{8}+e_{3} e_{6},-e_{1} e_{5}+e_{3} e_{7}, e_{1} e_{6}+e_{3} e_{8},-e_{1} e_{8}+e_{4} e_{5},-e_{1} e_{7}+e_{4} e_{6}, e_{1} e_{6}+e_{4} e_{7}, \\
& \left.e_{1} e_{5}+e_{4} e_{8}, e_{1} e_{2}+e_{5} e_{6}, e_{1} e_{3}+e_{5} e_{7},-e_{1} e_{4}+e_{5} e_{8},-e_{1} e_{4}+e_{6} e_{7},-e_{1} e_{3}+e_{6} e_{8}, e_{1} e_{2}+e_{7} e_{8}\right\}
\end{aligned}
$$

which generates a copy of $\mathfrak{s p i n}(7) \subset \mathfrak{s p i n}(8) \subset C l_{8}^{0}$, i.e.

$$
\{+1 \text { eigenspace of } \tau \star\} \cong \mathfrak{s p i n}(7) .
$$

By taking appropriate sums of these generators we can find the set of generators (9) of our copy of $\mathfrak{g}_{2}$. Moreover, $\mathfrak{g}_{2}$ is the intersection of the two copies of $\mathfrak{s p i n}(7)$, i.e.

$$
\mathfrak{g}_{2}=\mathfrak{s p i n}\left(\operatorname{span}\left\{e_{2}, \ldots, e_{8}\right\}\right) \cap\{+1 \text { eigenspace of } \tau \star\} .
$$


One can easlily compute brackets (in Clifford product) of the pair of Lie algebras ( $\mathfrak{s p i n}(7), \mathfrak{g}_{2}$ ) and check they form a symmetric pair. Since the orbit space $\operatorname{Spin}(7) \cdot e_{1}=\operatorname{Spin}(7) / G_{2}$ is 7 -dimensional and a submanifold of the 7-dimensional sphere $S^{7}=\operatorname{Spin}(8) / \operatorname{Spin}(7)$, we have the classical result [16]

$$
\frac{\operatorname{Spin}(7)}{G_{2}}=S^{7} .
$$

The 7 -dimensional eigenspace corresponding to -1 is generated by

$$
\begin{aligned}
& \left\{e_{1} e_{3}+e_{2} e_{4}-e_{5} e_{7}+e_{6} e_{8}, e_{1} e_{4}-e_{2} e_{3}+e_{5} e_{8}+e_{6} e_{7}, e_{1} e_{7}+e_{2} e_{8}-e_{3} e_{5}+e_{4} e_{6}, e_{1} e_{8}-e_{2} e_{7}+e_{3} e_{6}+e_{4} e_{5},\right. \\
& \left.-e_{1} e_{6}+e_{2} e_{5}+e_{3} e_{8}+e_{4} e_{7},-e_{1} e_{2}+e_{3} e_{4}+e_{5} e_{6}+e_{7} e_{8},-e_{1} e_{5}-e_{2} e_{6}-e_{3} e_{7}+e_{4} e_{8}\right\} .
\end{aligned}
$$

Remark. Note that, by using the bases

$$
\left\{e_{1} e_{2}, e_{1} e_{3}, \ldots, e_{7} e_{8}\right\} \subset \mathfrak{s p i n}(8)
$$

and

$$
\left\{E_{1,2}, E_{1,3}, \ldots, E_{7,8}\right\} \subset \mathfrak{s o}(8),
$$

the matrices representing

$$
\begin{aligned}
& \kappa_{8^{\star}}^{-}: \mathfrak{s p i n}(8) \longrightarrow \\
& \kappa_{8^{\star}}^{+}: \mathfrak{s p i n}(8) \longrightarrow \mathfrak{s o}(8), \\
& \quad \longrightarrow \quad s o(8),
\end{aligned}
$$

with respect to these bases equal the matrices representing $2 \sigma_{\star}$ and $2 \tau_{\star}$ respectively. In this way, triality becomes somewhat tautological.

\subsubsection{Group generated by $\sigma_{\star}$ and $\tau \star$}

Corollary 4.1. The endomorphisms $\sigma_{\star}$ and $\tau \star$ generate a copy of the permutation group $S_{3}$ of three symbols.

Proof. The endomorphisms $\sigma \star$ and $\tau \star$ satisfy

$$
\begin{aligned}
\tau_{\star}^{2} & =\operatorname{Id}_{\mathfrak{s p i n}(8)}, \\
\sigma_{\star}^{3} & =\operatorname{Id}_{\mathfrak{s p i n}(8)}, \\
\sigma_{\star} \tau_{\star} & =\tau_{\star} \sigma_{\star}^{2}, \\
\sigma_{\star}^{2} \tau_{\star} & =\tau_{\star} \sigma_{\star},
\end{aligned}
$$

which proves the claim.

Corollary 4.2. The compositions $\tau \star \sigma_{\star}, \sigma_{\star} \tau \star$ are also involutions.

Proof. Consider,

$$
\begin{aligned}
\left(\tau \star \sigma_{\star}\right)\left(\tau \star \sigma_{\star}\right) & =\tau \star\left(\sigma_{\star} \tau \star\right) \sigma_{\star} \\
& =\tau \star\left(\tau \star \sigma_{\star}^{2}\right) \sigma_{\star} \\
& =\tau_{\star}^{2} \sigma_{\star}^{3} \\
& =\operatorname{Id}_{\mathfrak{s p i n}(8)} .
\end{aligned}
$$


Corollary 4.3. The endomorphisms $\lambda_{8^{\star}}, \kappa_{8^{\star}}^{+}, \kappa_{8^{\star}}^{-}, \tau \star$ and $\sigma_{\star}$ satisfy

$$
\begin{aligned}
\lambda_{8^{\star}} \sigma_{\star} & =\kappa_{8^{\star}}^{-}, \\
\lambda_{8^{\star}} \tau_{\star} & =\kappa_{8^{\star}}^{+}, \\
\kappa_{8^{\star}}^{-} \tau_{\star} \sigma_{\star} & =\kappa_{8^{\star}}^{+}, \\
\kappa_{8^{\star}}^{+} \tau_{\star} \sigma_{\star} & =\kappa_{8^{\star}}^{-},
\end{aligned}
$$

i.e. the symmetric group $S_{3}$ generated by $\tau \star$ and $\sigma_{\star}$ permutes the Lie algebra representations $\lambda_{8^{\star}}, \kappa_{8^{\star}}^{+}$and $\kappa_{8^{\star}}^{-}$, and the following diagram commutes

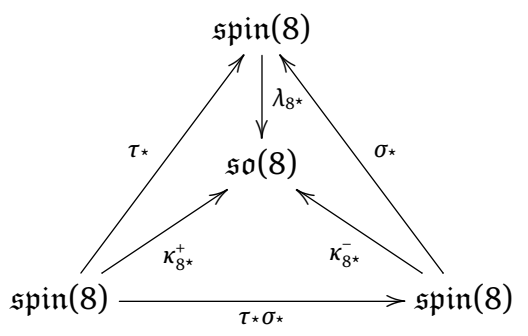

We know that the following diagrams also commute
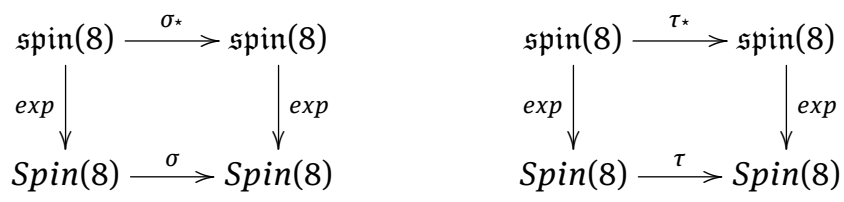

Corollary 4.4. The symmetric group $S_{3}$ generated by $\sigma$ and $\tau$ permutes the three representations $\lambda_{8}$, $\kappa_{8}^{+}$and $\kappa_{8}^{+}$, i.e. the following diagram commutes

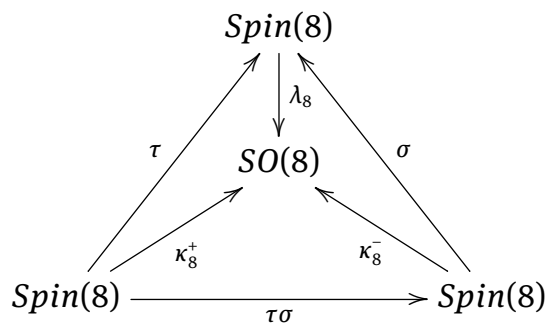

Corollary 4.5. We have

$\left\{(+1)\right.$-eigenspace of $\left.\tau \star \sigma_{\star}\right\} \cong \mathfrak{s p i n}\left(\operatorname{span}\left(e_{2}, e_{3}, e_{4}, e_{5}, e_{6}, e_{7}, e_{8}\right)\right)=\mathfrak{s p i n}(7)$.

Proof. It is enough to check the effect of $\tau \star \sigma_{\star}$ on the linear generators of $\mathfrak{s p i n}(8)$. We have

$$
\begin{aligned}
\tau \star \sigma_{\star}\left(e_{1} e_{k}\right) & =-e_{1} e_{k} & & \text { for } 2 \leq k \leq 8 . \\
\tau \star \sigma_{\star}\left(e_{i} e_{j}\right) & =e_{i} e_{j} & & \text { for } 2 \leq i<j \leq 8 .
\end{aligned}
$$

Corollary 4.6. We have

$$
\mathfrak{g}_{2}=\{(+1) \text {-eigenspace of } \tau \star\} \cap\left\{(+1) \text {-eigenspace of } \tau \star \sigma_{\star}^{2}\right\} \text {. }
$$


and

$$
\mathfrak{g}_{2}=\{(+1) \text {-eigenspace of } \tau \star\} \cap\left\{(+1) \text {-eigenspace of } \tau \star \sigma_{\star}\right\} \text {. }
$$

Proof. If $X \in\{(+1)$-eigenspace of $\tau \star\} \cap\left\{(+1)\right.$-eigenspace of $\left.\tau \star \sigma_{\star}^{2}\right\}$

$$
\begin{aligned}
\tau \star(X) & =X \\
\tau \star \sigma_{\star}^{2}(X) & =X,
\end{aligned}
$$

Then

$$
\begin{aligned}
X & =\tau \star \sigma_{\star}^{2}(X) \\
& =\sigma_{\star} \tau \star(X) \\
& =\sigma_{\star}(X),
\end{aligned}
$$

which means $X$ is a (+1)-eigenvector of $\sigma_{\star}$, thus an element of $\mathfrak{g}_{2}$. A dimension count proves the first identity. The second identity is proved similarly.

Corollary 4.7. $\sigma_{\star}^{2}$ provides an isomorphism between the two copies of $\mathfrak{s p i n}(7)$. Namely,

$$
\sigma_{\star}^{2}\left(\left\{(+1) \text {-eigenspace of } \tau \star \sigma_{\star}\right\}\right)=\{(+1) \text {-eigenspace of } \tau \star\}
$$

Proof. Let $Y \in\left\{(+1)\right.$-eigenspace of $\left.\tau \star \sigma_{\star}\right\}$, i.e.

$$
\tau \star \sigma_{\star}(Y)=Y
$$

Apply $\sigma_{\star}^{2}$ to both sides, so that

$$
\sigma_{\star}^{2} \tau \star \sigma_{\star}(Y)=\sigma_{\star}^{2}(Y),
$$

Since

$$
\sigma_{\star}^{2} \tau_{\star} \sigma_{\star}=\sigma_{\star}^{2} \tau_{\star} \sigma_{\star}^{2} \sigma_{\star}^{2}=\sigma_{\star}^{2} \sigma_{\star} \tau_{\star} \sigma_{\star}^{2}=\tau_{\star} \sigma_{\star}^{2}
$$

we have

$$
\tau \star\left(\sigma_{\star}^{2}(Y)\right)=\sigma_{\star}^{2}(Y) .
$$

This means that $X=\sigma_{\star}^{2}(Y)$ is a (+1)-eigenvector of $\tau \star$. Since $\tau \star$ is an automorphism, the claim is proved.

\subsubsection{Fundamental $\operatorname{Spin}(7)$ 4-form and $G_{2}$ 3-form}

Using the metric, we can dualize the endomorphisms $\kappa_{8^{\star}}^{+}\left(e_{i} e_{j}\right), 2 \leq i<j \leq 8$ into 2-forms:

$$
\begin{aligned}
& f_{2,3}=d x_{1} \wedge d x_{4}+d x_{2} \wedge d x_{3}+d x_{5} \wedge d x_{8}+d x_{6} \wedge d x_{7}, \\
& f_{2,4}=-d x_{1} \wedge d x_{3}+d x_{2} \wedge d x_{4}+d x_{5} \wedge d x_{7}-d x_{6} \wedge d x_{8}, \\
& f_{2,5}=d x_{1} \wedge d x_{6}+d x_{2} \wedge d x_{5}-d x_{3} \wedge d x_{8}-d x_{4} \wedge d x_{7}, \\
& f_{2,6}=-d x_{1} \wedge d x_{5}+d x_{2} \wedge d x_{6}-d x_{3} \wedge d x_{7}+d x_{4} \wedge d x_{8}, \\
& f_{2,7}=d x_{1} \wedge d x_{8}+d x_{2} \wedge d x_{7}+d x_{3} \wedge d x_{6}+d x_{4} \wedge d x_{5}, \\
& f_{2,8}=-d x_{1} \wedge d x_{7}+d x_{2} \wedge d x_{8}+d x_{3} \wedge d x_{5}-d x_{4} \wedge d x_{6}, \\
& f_{3,4}=d x_{1} \wedge d x_{2}+d x_{3} \wedge d x_{4}-d x_{5} \wedge d x_{6}-d x_{7} \wedge d x_{8}, \\
& f_{3,5}=d x_{1} \wedge d x_{7}+d x_{2} \wedge d x_{8}+d x_{3} \wedge d x_{5}+d x_{4} \wedge d x_{6}, \\
& f_{3,6}=-d x_{1} \wedge d x_{8}+d x_{2} \wedge d x_{7}+d x_{3} \wedge d x_{6}-d x_{4} \wedge d x_{5}, \\
& f_{3,7}=-d x_{1} \wedge d x_{5}-d x_{2} \wedge d x_{6}+d x_{3} \wedge d x_{7}+d x_{4} \wedge d x_{8},
\end{aligned}
$$




$$
\begin{aligned}
& f_{3,8}=d x_{1} \wedge d x_{6}-d x_{2} \wedge d x_{5}+d x_{3} \wedge d x_{8}-d x_{4} \wedge d x_{7}, \\
& f_{4,5}=-d x_{1} \wedge d x_{8}+d x_{2} \wedge d x_{7}-d x_{3} \wedge d x_{6}+d x_{4} \wedge d x_{5}, \\
& f_{4,6}=-d x_{1} \wedge d x_{7}-d x_{2} \wedge d x_{8}+d x_{3} \wedge d x_{5}+d x_{4} \wedge d x_{6}, \\
& f_{4,7}=d x_{1} \wedge d x_{6}-d x_{2} \wedge d x_{5}-d x_{3} \wedge d x_{8}+d x_{4} \wedge d x_{7}, \\
& f_{4,8}=d x_{1} \wedge d x_{5}+d x_{2} \wedge d x_{6}+d x_{3} \wedge d x_{7}+d x_{4} \wedge d x_{8}, \\
& f_{5,6}=d x_{1} \wedge d x_{2}-d x_{3} \wedge d x_{4}+d x_{5} \wedge d x_{6}-d x_{7} \wedge d x_{8}, \\
& f_{5,7}=d x_{1} \wedge d x_{3}+d x_{2} \wedge d x_{4}+d x_{5} \wedge d x_{7}+d x_{6} \wedge d x_{8}, \\
& f_{5,8}=-d x_{1} \wedge d x_{4}+d x_{2} \wedge d x_{3}+d x_{5} \wedge d x_{8}-d x_{6} \wedge d x_{7}, \\
& f_{6,7}=-d x_{1} \wedge d x_{4}+d x_{2} \wedge d x_{3}-d x_{5} \wedge d x_{8}+d x_{6} \wedge d x_{7}, \\
& f_{6,8}=-d x_{1} \wedge d x_{3}-d x_{2} \wedge d x_{4}+d x_{5} \wedge d x_{7}+d x_{6} \wedge d x_{8}, \\
& f_{7,8}=d x_{1} \wedge d x_{2}-d x_{3} \wedge d x_{4}-d x_{5} \wedge d x_{6}+d x_{7} \wedge d x_{8} .
\end{aligned}
$$

We can form the Spin(7)-invariant 4-form

$$
\begin{aligned}
\Omega= & \sum_{2 \leq i<j \leq 8} f_{i, j} \wedge f_{i, j} \\
= & 6\left[-d x_{1} \wedge d x_{2} \wedge d x_{3} \wedge d x_{4}+d x_{1} \wedge d x_{4} \wedge d x_{6} \wedge d x_{7}-d x_{1} \wedge d x_{2} \wedge d x_{5} \wedge d x_{6}\right. \\
& -d x_{1} \wedge d x_{3} \wedge d x_{5} \wedge d x_{7}-d x_{1} \wedge d x_{2} \wedge d x_{7} \wedge d x_{8}+d x_{1} \wedge d x_{3} \wedge d x_{6} \wedge d x_{8} \\
& +d x_{1} \wedge d x_{4} \wedge d x_{5} \wedge d x_{8}+d x_{2} \wedge d x_{4} \wedge d x_{5} \wedge d x_{7}-d x_{2} \wedge d x_{4} \wedge d x_{6} \wedge d x_{8} \\
& +d x_{2} \wedge d x_{3} \wedge d x_{5} \wedge d x_{8}+d x_{2} \wedge d x_{3} \wedge d x_{6} \wedge d x_{7}-d x_{3} \wedge d x_{4} \wedge d x_{5} \wedge d x_{6} \\
& \left.-d x_{3} \wedge d x_{4} \wedge d x_{7} \wedge d x_{8}-d x_{5} \wedge d x_{6} \wedge d x_{7} \wedge d x_{8}\right]
\end{aligned}
$$

whose square is a multiple of the 8-dimensional volume form

$$
\Omega \wedge \Omega=504 d x_{1} \wedge d x_{2} \wedge d x_{3} \wedge d x_{4} \wedge d x_{5} \wedge d x_{6} \wedge d x_{7} \wedge d x_{8},
$$

thus showing that $\Omega$ is non-degenerate.

By integrating out $d x_{1}$ we get the $G_{2}$-invariant 3-form

$$
\begin{aligned}
\varphi= & 6\left(-\left(d x_{2} \wedge d x_{3} \wedge d x_{4}\right)-\left(d x_{2} \wedge d x_{5} \wedge d x_{6}\right)-\left(d x_{2} \wedge d x_{7} \wedge d x_{8}\right)\right. \\
& \left.-\left(d x_{3} \wedge d x_{5} \wedge d x_{7}\right)+\left(d x_{4} \wedge d x_{6} \wedge d x_{7}\right)+\left(d x_{3} \wedge d x_{6} \wedge d x_{8}\right)+\left(d x_{4} \wedge d x_{5} \wedge d x_{8}\right)\right) .
\end{aligned}
$$

\subsection{6 $\sigma$ and $\tau$ are outer automorphisms}

Now, we will show that $\sigma$ and $\tau$ are outer automorphisms by showing that they permute the non-trivial central elements of $\operatorname{Spin}(8)$, namely

$$
-1, \mathrm{vol}_{8},-\mathrm{vol}_{8} \text {. }
$$

Recall that

$$
\operatorname{vol}_{8}^{2}=1
$$

Consider, for instance,

$$
\begin{aligned}
\sigma\left(\exp \left(t e_{1} e_{2}\right)\right) & =\sigma\left(\cos (t)+\sin (t) e_{1} e_{2}\right) \\
& =\exp \left(\sigma_{\star}\left(t e_{1} e_{2}\right)\right) \\
& =\exp \left(\frac{t}{2}\left(-e_{1} e_{2}-e_{3} e_{4}-e_{5} e_{6}-e_{7} e_{8}\right)\right) \\
& =\exp \left(-\frac{t e_{1} e_{2}}{2}\right) \exp \left(-\frac{t e_{3} e_{4}}{2}\right) \exp \left(-\frac{t e_{5} e_{6}}{2}\right) \exp \left(-\frac{t e_{7} e_{8}}{2}\right)
\end{aligned}
$$




$$
=\left(\cos (t / 2)-\sin (t / 2) e_{1} e_{2}\right)\left(\cos (t / 2)-\sin (t / 2) e_{3} e_{4}\right)\left(\cos (t / 2)-\sin (t / 2) e_{5} e_{6}\right)\left(\cos (t / 2)-\sin (t / 2) e_{7} e_{8}\right) \text {, }
$$

so that

$$
\begin{aligned}
\sigma\left(e_{1} e_{2}\right) & =\sigma\left(\cos (\pi / 2)+\sin (\pi / 2) e_{1} e_{2}\right) \\
& =\left(\cos (\pi / 4)-\sin (\pi / 4) e_{1} e_{2}\right)\left(\cos (\pi / 4)-\sin (\pi / 4) e_{3} e_{4}\right)\left(\cos (\pi / 4)-\sin (\pi / 4) e_{5} e_{6}\right)\left(\cos (\pi / 4)-\sin (\pi / 4) e_{7} e_{8}\right) .
\end{aligned}
$$

Note that the calculations are carried out in $\mathrm{Cl}_{8}$ where the exponentials converge. Similarly,

$\sigma\left(e_{3} e_{4}\right)=\left(\cos (\pi / 4)+\sin (\pi / 4) e_{1} e_{2}\right)\left(\cos (\pi / 4)+\sin (\pi / 4) e_{3} e_{4}\right)\left(\cos (\pi / 4)-\sin (\pi / 4) e_{5} e_{6}\right)\left(\cos (\pi / 4)-\sin (\pi / 4) e_{7} e_{8}\right)$

$\sigma\left(e_{5} e_{6}\right)=\left(\cos (\pi / 4)+\sin (\pi / 4) e_{1} e_{2}\right)\left(\cos (\pi / 4)-\sin (\pi / 4) e_{3} e_{4}\right)\left(\cos (\pi / 4)+\sin (\pi / 4) e_{5} e_{6}\right)\left(\cos (\pi / 4)-\sin (\pi / 4) e_{7} e_{8}\right)$.

$\sigma\left(e_{7} e_{8}\right)=\left(\cos (\pi / 4)+\sin (\pi / 4) e_{1} e_{2}\right)\left(\cos (\pi / 4)-\sin (\pi / 4) e_{3} e_{4}\right)\left(\cos (\pi / 4)-\sin (\pi / 4) e_{5} e_{6}\right)\left(\cos (\pi / 4)+\sin (\pi / 4) e_{7} e_{8}\right)$.

Now consider

$$
\begin{aligned}
\tau\left(\exp \left(t e_{1} e_{2}\right)\right) & =\tau\left(\cos (t)+\sin (t) e_{1} e_{2}\right) \\
& =\exp \left(\tau \star\left(t e_{1} e_{2}\right)\right) \\
& =\exp \left(\frac{t}{2}\left(e_{1} e_{2}+e_{3} e_{4}+e_{5} e_{6}+e_{7} e_{8}\right)\right) \\
& =\exp \left(\frac{t e_{1} e_{2}}{2}\right) \exp \left(\frac{t e_{3} e_{4}}{2}\right) \exp \left(\frac{t e_{5} e_{6}}{2}\right) \exp \left(\frac{t e_{7} e_{8}}{2}\right) \\
& =\left(\cos (t / 2)+\sin (t / 2) e_{1} e_{2}\right)\left(\cos (t / 2)+\sin (t / 2) e_{3} e_{4}\right)\left(\cos (t / 2)+\sin (t / 2) e_{5} e_{6}\right)\left(\cos (t / 2)+\sin (t / 2) e_{7} e_{8}\right),
\end{aligned}
$$

so that

$$
\begin{aligned}
\tau\left(e_{1} e_{2}\right) & =\tau\left(\cos (\pi / 2)+\sin (\pi / 2) e_{1} e_{2}\right) \\
& =\left(\cos (\pi / 4)+\sin (\pi / 4) e_{1} e_{2}\right)\left(\cos (\pi / 4)+\sin (\pi / 4) e_{3} e_{4}\right)\left(\cos (\pi / 4)+\sin (\pi / 4) e_{5} e_{6}\right)\left(\cos (\pi / 4)+\sin (\pi / 4) e_{7} e_{8}\right) .
\end{aligned}
$$

Similarly for all other generators $e_{i} e_{j}$ of $\mathfrak{s p i n}(8) \subset C l_{8}^{0}$.

Corollary 4.8. The automorphisms $\tau$ and $\sigma$ are outer automorphisms, of order 2 and 3 respectively, since they permute the elements of the center of Spin(8), i.e.

$$
\begin{aligned}
\sigma(-1) & =\mathrm{vol}_{8}, \\
\sigma\left(\mathrm{vol}_{8}\right) & =-\mathrm{vol}_{8}, \\
\sigma\left(-\mathrm{vol}_{8}\right) & =-1 \\
\tau(-1) & =\mathrm{vol}_{8}, \\
\tau\left(\mathrm{vol}_{8}\right) & =-1
\end{aligned}
$$

Proof. Consider

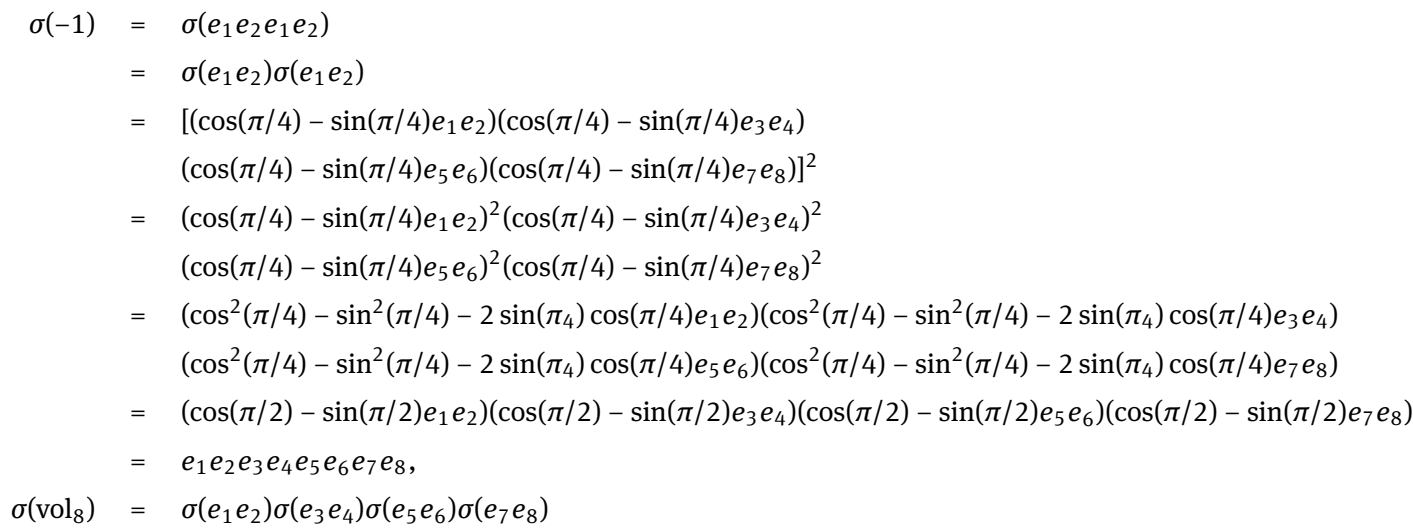




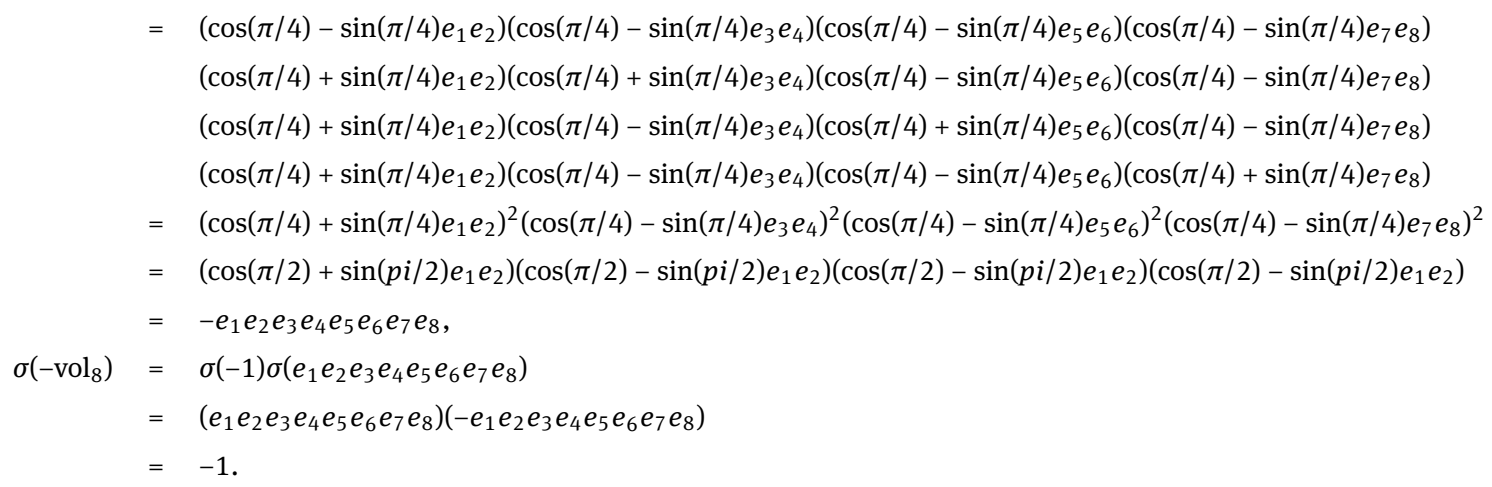

On the other hand, we also have

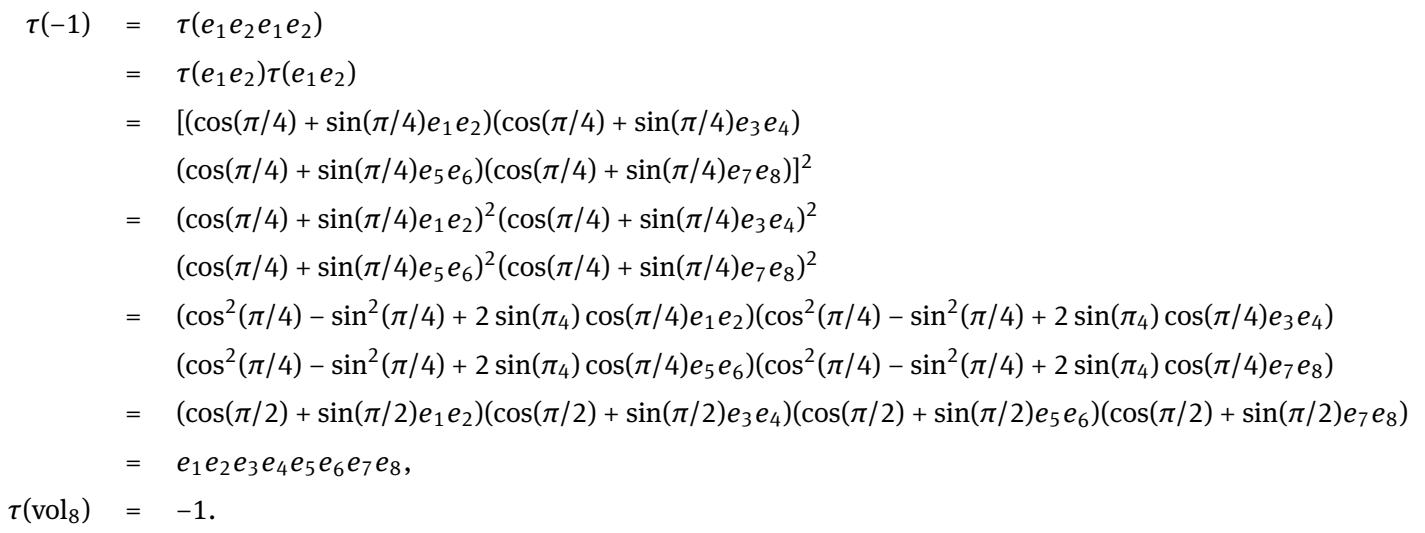

\subsubsection{Octonions}

The relationship between Spin(8) and the Octonions is well known (see [4, 17]). In this subsection, as an example of the use of the binary encoding, we recover a multiplication table of the normed division algebra of Octonions using the Spin(8) representations. The idea is to consider Clifford multiplication and the three real representations of $\operatorname{Spin}(8)$ at the same time. We follow [4] and consider Clifford multiplication as a trilinear map (a "triality" as defined by Adams in [1]) and dualization to get a bilinear map $\mathbb{R}^{8} \times \tilde{\Delta}_{8}^{+} \rightarrow \tilde{\Delta}_{8}^{-}$. By identifying the three spaces with a single space (in a suitable way) one can define a product on it using this bilinear map.

Consider the basis of positive real spinors given by

$$
\beta^{+}=\left\{u_{0}-u_{15}, i u_{0}+i u_{15}, u_{3}+u_{12},-i u_{3}+i u_{12},-u_{5}+u_{10}, i u_{5}+i u_{10}, u_{6}+u_{9}, i u_{6}-i u_{9}\right\} \subset \tilde{\Delta}_{8}^{+}
$$

Let us consider Clifford multiplication as a bilinear map

$$
\mathbb{R}^{8} \times \tilde{\Delta}_{8}^{+} \rightarrow \tilde{\Delta}_{8}^{-} .
$$


For this subsection, let us denote $\left\{v_{0}, \ldots, v_{7}\right\}$ the standard ordered basis of $\mathbb{R}^{8}$. The Clifford multiplication table is the following:

\begin{tabular}{|r|rrrrrrrr|}
\hline & $u_{0}-u_{15}$ & $i u_{0}+i u_{15}$ & $u_{3}+u_{12}$ & $-i u_{3}+i u_{12}$ & $-u_{5}+u_{10}$ & $i u_{5}+i u_{10}$ & $u_{6}+u_{9}$ & $i u_{6}-i u_{9}$ \\
\hline$v_{0}$ & $i u_{1}-i u_{14}$ & $-u_{1}-u_{14}$ & $i u_{2}+i u_{13}$ & $-u_{2}+u_{13}$ & $i u_{4}-i u_{11}$ & $-u_{4}-u_{11}$ & $i u_{7}+i u_{8}$ & $-u_{7}+u_{8}$ \\
$v_{1}$ & $u_{1}+u_{14}$ & $i u_{1}-i u_{14}$ & $-u_{2}+u_{13}$ & $-i u_{2}-i u_{13}$ & $-u_{4}-u_{11}$ & $-i u_{4}+i u_{11}$ & $u_{7}-u_{8}$ & $i u_{7}+i u_{8}$ \\
$v_{2}$ & $-i u_{2}-i u_{13}$ & $u_{2}-u_{13}$ & $i u_{1}-i u_{14}$ & $-u_{1}-u_{14}$ & $i u_{7}+i u_{8}$ & $-u_{7}+u_{8}$ & $-i u_{4}+i u_{11}$ & $u_{4}+u_{11}$ \\
$v_{3}$ & $-u_{2}+u_{13}$ & $-i u_{2}-i u_{13}$ & $-u_{1}-u_{14}$ & $-i u_{1}+i u_{14}$ & $u_{7}-u_{8}$ & $i u_{7}+i u_{8}$ & $u_{4}+u_{11}$ & $i u_{4}-i u_{11}$ \\
$v_{4}$ & $i u_{4}-i u_{11}$ & $-u_{4}-u_{11}$ & $i u_{7}+i u_{8}$ & $-u_{7}+u_{8}$ & $-i u_{1}+i u_{14}$ & $u_{1}+u_{14}$ & $-i u_{2}-i u_{13}$ & $u_{2}-u_{13}$ \\
$v_{5}$ & $u_{4}+u_{11}$ & $i u_{4}-i u_{11}$ & $u_{7}-u_{8}$ & $i u_{7}+i u_{8}$ & $u_{1}+u_{14}$ & $i u_{1}-i u_{14}$ & $u_{2}-u_{13}$ & $i u_{2}+i u_{13}$ \\
$v_{6}$ & $-i u_{8}-i u_{7}$ & $u_{8}-u_{7}$ & $-i u_{11}+i u_{4}$ & $u_{11}+u_{4}$ & $-i u_{13}-i u_{2}$ & $u_{13}-u_{2}$ & $-i u_{14}+i u_{1}$ & $u_{14}+u_{1}$ \\
$v_{7}$ & $-u_{8}+u_{7}$ & $-i u_{8}-i u_{7}$ & $-u_{11}-u_{4}$ & $-i u_{11}+i u_{4}$ & $-u_{13}+u_{2}$ & $-i u_{13}-i u_{2}$ & $-u_{14}-u_{1}$ & $-i u_{14}+i u_{1}$ \\
\hline
\end{tabular}

Now let

$$
\beta^{-}=\left\{i u_{1}-i u_{14},-u_{1}-u_{14}, i u_{2}+i u_{13},-u_{2}+u_{13}, i u_{4}-i u_{11},-u_{4}-u_{11}, i u_{7}+i u_{8},-u_{7}+u_{8}\right\} .
$$

By labeling the elements of the ordered bases $\beta^{+}=\left\{\psi_{0}, \ldots, \psi_{7}\right\}$ and $\beta^{-}=\left\{\phi_{0}, \ldots, \phi_{7}\right\}$, the Clifford multiplication table now reads as follows:

\begin{tabular}{|l|rrrrrrrr|}
\hline & $\psi_{0}$ & $\psi_{1}$ & $\psi_{2}$ & $\psi_{3}$ & $\psi_{4}$ & $\psi_{5}$ & $\psi_{6}$ & $\psi_{7}$ \\
\hline$v_{0}$ & $\phi_{0}$ & $\phi_{1}$ & $\phi_{2}$ & $\phi_{3}$ & $\phi_{4}$ & $\phi_{5}$ & $\phi_{6}$ & $\phi_{7}$ \\
$v_{1}$ & $-\phi_{1}$ & $\phi_{0}$ & $\phi_{3}$ & $-\phi_{2}$ & $\phi_{5}$ & $-\phi_{4}$ & $-\phi_{7}$ & $\phi_{6}$ \\
$v_{2}$ & $-\phi_{2}$ & $-\phi_{3}$ & $\phi_{0}$ & $\phi_{1}$ & $\phi_{6}$ & $\phi_{7}$ & $-\phi_{4}$ & $-\phi_{5}$ \\
$v_{3}$ & $\phi_{3}$ & $-\phi_{2}$ & $\phi_{1}$ & $-\phi_{0}$ & $-\phi_{7}$ & $\phi_{6}$ & $-\phi_{5}$ & $\phi_{4}$ \\
$v_{4}$ & $\phi_{4}$ & $\phi_{5}$ & $\phi_{6}$ & $\phi_{7}$ & $-\phi_{0}$ & $-\phi_{1}$ & $-\phi_{2}$ & $-\phi_{3}$ \\
$v_{5}$ & $-\phi_{5}$ & $\phi_{4}$ & $-\phi_{7}$ & $\phi_{6}$ & $-\phi_{1}$ & $\phi_{0}$ & $-\phi_{3}$ & $\phi_{2}$ \\
$v_{6}$ & $-\phi_{6}$ & $\phi_{7}$ & $\phi_{4}$ & $-\phi_{5}$ & $-\phi_{2}$ & $\phi_{3}$ & $\phi_{0}$ & $-\phi_{1}$ \\
$v_{7}$ & $-\phi_{7}$ & $-\phi_{6}$ & $\phi_{5}$ & $\phi_{4}$ & $-\phi_{3}$ & $-\phi_{2}$ & $\phi_{1}$ & $\phi_{0}$ \\
\hline
\end{tabular}

We can recover the multiplication table of the octonions by identifying $\mathbb{R}^{8}, \tilde{\Delta}_{8}^{+}$and $\tilde{\Delta}_{8}^{-}$with a single vector space $\mathbb{O}=\operatorname{span}\left\{\hat{e}_{0}, \hat{e}_{1}, \ldots, \hat{e}_{7}\right\}$ in the following way. We identify $v_{0}, \psi_{0}$ and $\phi_{0}$ with the identity $\hat{e}_{0}$ of $\mathbb{O}$. We also identify $\psi_{i}$ with $\hat{e}_{i}$. In this way, we have that $\phi_{i}=v_{0} \psi_{i}=\hat{e}_{0} \cdot \hat{e}_{i}=\hat{e}_{i}$. We also have that $v_{1} \psi_{0}=-\phi_{1}$ so that $v_{1}$ should be identify with $-\hat{e}_{1}$. In the same way $v_{2}, v_{3}, v_{4}, v_{5}, v_{6}$ and $v_{7}$ should be identify with $-\hat{e}_{2}, \hat{e}_{3}$ $\hat{e}_{4},-\hat{e}_{5},-\hat{e}_{6}$ and $-\hat{e}_{7}$. Then the multiplication table (of the octonions) reads as follows

\begin{tabular}{|c|rrrrrrrr|}
\hline & $\hat{e}_{0}$ & $\hat{e}_{1}$ & $\hat{e}_{2}$ & $\hat{e}_{3}$ & $\hat{e}_{4}$ & $\hat{e}_{5}$ & $\hat{e}_{6}$ & $\hat{e}_{7}$ \\
\hline$\hat{e}_{0}$ & $\hat{e}_{0}$ & $\hat{e}_{1}$ & $\hat{e}_{2}$ & $\hat{e}_{3}$ & $\hat{e}_{4}$ & $\hat{e}_{5}$ & $\hat{e}_{6}$ & $\hat{e}_{7}$ \\
$\hat{e}_{1}$ & $\hat{e}_{1}$ & $-\hat{e}_{0}$ & $-\hat{e}_{3}$ & $\hat{e}_{2}$ & $-\hat{e}_{5}$ & $\hat{e}_{4}$ & $\hat{e}_{7}$ & $-\hat{e}_{6}$ \\
$\hat{e}_{2}$ & $\hat{e}_{2}$ & $\hat{e}_{3}$ & $-\hat{e}_{0}$ & $-\hat{e}_{1}$ & $-\hat{e}_{6}$ & $-\hat{e}_{7}$ & $\hat{e}_{4}$ & $\hat{e}_{5}$ \\
$\hat{e}_{3}$ & $\hat{e}_{3}$ & $-\hat{e}_{2}$ & $\hat{e}_{1}$ & $-\hat{e}_{0}$ & $-\hat{e}_{7}$ & $\hat{e}_{6}$ & $-\hat{e}_{5}$ & $\hat{e}_{4}$ \\
$\hat{e}_{4}$ & $\hat{e}_{4}$ & $\hat{e}_{5}$ & $\hat{e}_{6}$ & $\hat{e}_{7}$ & $-\hat{e}_{0}$ & $-\hat{e}_{1}$ & $-\hat{e}_{2}$ & $-\hat{e}_{3}$ \\
$\hat{e}_{5}$ & $\hat{e}_{5}$ & $-\hat{e}_{4}$ & $\hat{e}_{7}$ & $-\hat{e}_{6}$ & $\hat{e}_{1}$ & $-\hat{e}_{0}$ & $\hat{e}_{3}$ & $-\hat{e}_{2}$ \\
$\hat{e}_{6}$ & $\hat{e}_{6}$ & $-\hat{e}_{7}$ & $-\hat{e}_{4}$ & $\hat{e}_{5}$ & $\hat{e}_{2}$ & $-\hat{e}_{3}$ & $-\hat{e}_{0}$ & $\hat{e}_{1}$ \\
$\hat{e}_{7}$ & $\hat{e}_{7}$ & $\hat{e}_{6}$ & $-\hat{e}_{5}$ & $-\hat{e}_{4}$ & $\hat{e}_{3}$ & $\hat{e}_{2}$ & $-\hat{e}_{1}$ & $-\hat{e}_{0}$ \\
\hline
\end{tabular}

Remark. One can actually do the same in the case of $\mathbb{R}^{4}, \tilde{\Delta}_{4}^{+}$and $\tilde{\Delta}_{4}^{-}$to recover the quaternion multiplication table.

\subsection{Vector fields on spheres}

Classical results of Hurwitz, Radon and Adams [2,15] tell us the maximal number of independent vector fields a sphere can admit, which is given in terms of the Hurwitz Radon numbers. In this subsection, we will give 
explicit expressions, using Clifford algebras and the binary code, for a maximal set of orthogonal linearly independent vector fields on spheres (compare with $[19,20]$ for recent work). We follow the ideas described in $[14,16]$ using Clifford multiplication, but we will multiply by elements of $\mathfrak{s p i n}(r)$ instead of multiplying by elements of the standard basis of $\mathbb{R}^{r}$.

The idea is as follows: Let $N \in \mathbb{N}$ and suppose that $\mathbb{R}^{N}$ is a non-trivial representation of $C l_{r}^{0}$ (not necessarily irreducible). Since Clifford multiplication by unit vectors is an orthogonal transformation on the space of real spinors, for every $Z \in S^{N-1}$ and $2 \leq j \leq r$, the $r-1$ vectors $V_{j-1}(Z):=e_{1} e_{j} Z$ form an orthonormal set tangent to the sphere at $Z$. By [2], if $r$ is the maximum integer such that $\mathbb{R}^{N}$ is a non-trivial representation of $\mathrm{Cl}_{r}^{0}$, then the set of independent vector fields is maximal. Although the calculations below may seem cumbersome due to the slightly more complicated form of the basic vectors of the real Spin representations [3], the main point is that our expressions provide a general way to produce explicitly the vector fields. As a concrete example, we compute the 9 vector fields on $S^{31}$.

Thus, let us suppose that $C l_{r}^{0}$ is represented on $\mathbb{R}^{N}$, for some $N \in \mathbb{N}$, in such a way that each bivector $e_{i} e_{j}$ is mapped to an antisymmetric endomorphism $J_{i j}$ satisfying

$$
J_{i j}^{2}=-\mathrm{Id}_{\mathbb{R}^{N}} .
$$

- If $r \not \equiv 0(\bmod 4), r>1, \mathbb{R}^{N}$ decomposes into a sum of irreducible representations of $C l_{r}^{0}$. Since this algebra is simple, such irreducible representations can only be trivial or copies of the standard representation $\tilde{\Delta}_{r}$ of $\mathrm{Cl}_{r}^{0}$ (cf. [16]). Due to (10), there are no trivial summands in such a decomposition so that

$$
\mathbb{R}^{N}=\underbrace{\tilde{\Delta}_{r} \oplus \cdots \oplus \tilde{\Delta}_{r}}_{m \text { times }} .
$$

By restricting to $\mathfrak{s p i n}(r) \subset C l_{r}^{0}$,

$$
\mathbb{R}^{N}=\tilde{\Delta}_{r} \otimes_{\mathbb{R}} \mathbb{R}^{m}
$$

we see that $\mathfrak{s p i n}(r)$ has an isomorphic image

$$
\widehat{\mathfrak{s p i n}(r)}=\mathfrak{s p i n}(r) \otimes\left\{\operatorname{Id}_{\mathbb{R}^{m}}\right\} \subset \mathfrak{s o}\left(d_{r} m\right),
$$

which is a subalgebra of $\mathfrak{s o}\left(d_{r} m\right)$. Note that

$$
J_{i j}=\left[\kappa_{r}\left(e_{i} e_{j}\right) \otimes \operatorname{Id}_{\mathbb{R}^{m}}\right]
$$

for $1 \leq i<j \leq r$.

- If, on the other hand, $r \equiv 0(\bmod 4)$,

$$
\hat{\Delta}_{r}=\tilde{\Delta}_{r}^{+} \oplus \tilde{\Delta}_{r}^{-},
$$

the sum of two inequivalent irreducible representationsm, and

$$
\mathbb{R}^{N}=\tilde{\Delta}_{r}^{+} \otimes_{\mathbb{R}} \mathbb{R}^{m_{1}} \oplus \tilde{\Delta}_{r}^{-} \otimes_{\mathbb{R}} \mathbb{R}^{m_{2}} .
$$

as a representation to $\mathfrak{s p i n}(r) \subset C l_{r}^{0}$, and we see that $\mathfrak{s p i n}(r)$ has an isomorphic image

$$
\widehat{\mathfrak{s p i n}(r)}=\left\{\boldsymbol{\kappa}_{n}^{+}(g) \otimes\left(\operatorname{Id}_{\mathbb{R}^{m_{1}}} \oplus \mathbf{0}_{m_{2} \times m_{2}}\right) \oplus \boldsymbol{\kappa}_{n}^{-}(g) \otimes\left(\mathbf{0}_{m_{1} \times m_{1}} \oplus \operatorname{Id}_{\mathbb{R}^{m_{2}}}\right) \mid g \in \mathfrak{s p i n}(r)\right\} \subset \mathfrak{s o}\left(d_{r} m_{1}+d_{r} m_{2}\right) .
$$

Note that

$$
J_{i j}=\left[\kappa_{r}^{+}\left(e_{i} e_{j}\right) \otimes \operatorname{Id}_{\mathbb{R}^{m_{1}}}\right] \oplus\left[\kappa_{r}^{-}\left(e_{i} e_{j}\right) \otimes \operatorname{Id}_{\mathbb{R}^{m_{2}}}\right]
$$

for $1 \leq i<j \leq r$.

Given a point $Z$ in the sphere of $\tilde{\Delta}_{r} \otimes_{\mathbb{R}} \mathbb{R}^{m}$ or $\tilde{\Delta}_{r}^{ \pm} \otimes_{\mathbb{R}} \mathbb{R}^{m}$, the corresponding values of the vector fields at $Z$ will be given by

$$
\left[\kappa_{r}\left(e_{1} e_{2}\right) \otimes \operatorname{Id}_{m}\right](Z), \ldots,\left[\kappa_{r}\left(e_{1} e_{r}\right) \otimes \operatorname{Id}_{m}\right](Z),
$$

or

$$
\left[\kappa_{r}^{ \pm}\left(e_{1} e_{2}\right) \otimes \operatorname{Id}_{m}\right](Z), \ldots,\left[\kappa_{r}^{ \pm}\left(e_{1} e_{r}\right) \otimes \operatorname{Id}_{m}\right](Z),
$$

respectively, where $\operatorname{Id}_{m}:=\operatorname{Id}_{\mathbb{R}^{m}}$. 


\subsubsection{Calculations in $\Delta_{r}$}

First recall that

$$
e_{1} u_{b}=i u_{b+(-1)^{b_{0}}}
$$

Now, if $p=2 \leq r, j=\left[\frac{p+1}{2}\right]=1$,

$$
e_{1} e_{2} \cdot u_{a}=i(-1)^{a_{0}} u_{a}
$$

if $3 \leq p \leq 2\left[\frac{r}{2}\right], j=\left[\frac{p+1}{2}\right] \geq 2$,

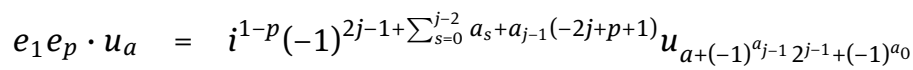

and if $p=r=2 k+1$,

$$
e_{1} e_{r} u_{a}=(-1)^{[r / 2]+1+\sum_{l=0}^{[r / 2]-1} a_{l}} u_{a+(-1)^{a} 0} .
$$

We also have the following expressions for the real and quaternionic structures: For $r=0,1,4,5(\bmod 8)$ and $q=[r / 4]$

$$
\gamma_{r}\left(u_{a}\right)=(-i)^{q}(-1)^{\sum_{t=1}^{q} a_{2 t-1}} u_{2^{2 q}-1-a}
$$

For $r=2,3,6,7(\bmod 8)$ and $q=[r / 4]$

$$
\gamma_{r}\left(u_{a}\right)=(-i)^{q+1}(-1)^{\sum_{t=0}^{q} a_{2 t}} u_{2^{2 q+1}-1-a} .
$$

\subsubsection{The vector fields}

Due to the coincidence of dimensions of the real Spin representations

$$
d_{8 q+3}=d_{8 q+4}
$$

and

$$
d_{8 q+5}=d_{8 q+6}=d_{8 q+7}=d_{8 q+8},
$$

we only need to consider the cases $r \equiv 0,1,2,4(\bmod 8)$. For example, if $R^{N}$ is a representation space of $\mathrm{Cl}_{8 q+3}^{0}$, then $\mathrm{R}^{N}$ is also a representation of $\mathrm{Cl}_{8 q+4}^{0}$ and therefore $8 q+3$ is not maximal. Let us consider bases for the spaces $\tilde{\Delta}_{r}$ and $\tilde{\Delta}_{r}^{ \pm}$:

- $\quad$ Case $r \equiv 0(\bmod 8)$ : A basis for $\tilde{\Delta}_{r}^{+}$is given by

$$
\left\{u_{a}+\gamma_{r}\left(u_{a}\right), i u_{a}+\gamma_{r}\left(i u_{a}\right) \mid a=0, \ldots, 2^{r / 2-1}-1, \sum a_{l} \equiv 0(\bmod 2)\right\}
$$

- $\quad$ Case $r \equiv 1(\bmod 8)$ : A basis for $\tilde{\Delta}_{r}$ is given by

$$
\left\{u_{a}+\gamma_{r}\left(u_{a}\right), i u_{a}+\gamma_{r}\left(i u_{a}\right) \mid a=0, \ldots, 2^{[r / 2]-1}-1\right\}
$$

- $\quad$ Case $r \equiv 2(\bmod 8):$ A basis for $\tilde{\Delta}_{r}$ is given by

$$
\left\{u_{a}, i u_{a} \mid a=0, \ldots, 2^{r / 2}-1, \sum a_{l} \equiv 0(\bmod 2)\right\}
$$

- $\quad$ Case $r \equiv 4(\bmod 8)$ : A basis for $\tilde{\Delta}_{r}^{+}$is given by

$$
\left\{u_{a}, i u_{a} \mid a=0, \ldots, 2^{r / 2}-1, \sum a_{l} \equiv 0(\bmod 2)\right\}
$$




\section{Case $\boldsymbol{r} \equiv \mathbf{0}(\bmod \mathbf{8})$}

For any point $Z \in S\left(\tilde{\Delta}_{r}^{+} \otimes_{\mathbb{R}} \mathbb{R}^{m}\right)=S^{d_{r} m}$,

$$
Z=\sum_{h=1}^{m} \sum_{\left\{a=0, \ldots, 2^{r / 2-1}-1: \sum a_{l} \equiv 0(\bmod 2)\right\}} X_{a, h}\left(u_{a} \otimes v_{h}\right)+Y_{a, h}\left(i u_{a} \otimes v_{h}\right),
$$

where $\left\{v_{1}, \ldots, v_{m}\right\}$ is an orthonormal basis of $\mathbb{R}^{m}$ and $X_{a, h}, Y_{a, h} \in \mathbb{R}$, there are $r-1$ point-wise linearly independent vector fields given as follows. For $p=2$,

$$
\begin{aligned}
& {\left.\left.\left[\kappa_{r}\left(e_{1} e_{2}\right) \otimes \operatorname{Id}_{m}\right]\left(\sum_{h=1}^{m} \sum_{\left\{a=0, \ldots, 2^{r / 2-1}-1, \sum a_{l} \equiv 0(\bmod 2)\right\}} X_{a, h}\left(u_{a}+\gamma_{r}\left(u_{a}\right)\right) \otimes v_{h}\right)+Y_{a, h}\left(i u_{a}+\gamma_{r}\left(i u_{a}\right)\right) \otimes v_{h}\right)\right) } \\
= & \left.\left.\sum_{h=1}^{m} \sum_{\left\{a=0, \ldots, r^{r / 2-1}-1, \sum a_{l} \equiv 0(\bmod 2)\right\}}(-1)^{a_{0}}\left(-Y_{a, h}\left(u_{a}+\gamma_{r}\left(u_{a}\right)\right) \otimes v_{h}\right)+X_{a, h}\left(i u_{a}+\gamma_{r}\left(i u_{a}\right)\right) \otimes v_{h}\right)\right) .
\end{aligned}
$$

For $3 \leq p \leq r$,

$$
\begin{aligned}
& {\left.\left.\left[\kappa_{r}\left(e_{1} e_{p}\right) \otimes \operatorname{Id}_{m}\right]\left(\sum_{\left\{a=0, \ldots, 2^{r / 2-1}-1, \sum a_{l} \equiv 0(\bmod 2)\right\}} X_{a, h}\left(u_{a}+\gamma_{r}\left(u_{a}\right)\right) \otimes v_{h}\right)+Y_{a, h}\left(i u_{a}+\gamma_{r}\left(i u_{a}\right)\right) \otimes v_{h}\right)\right) } \\
= & \sum_{h=1}^{m} \sum_{\left\{a=0, \ldots, 2^{r / 2-1}-1, \sum a_{l} \equiv 0(\bmod 2)\right\}}(-1)^{2 j-1+\sum_{s=0}^{j-2} a_{s}+a_{j-1}(-2 j+p+1)} \\
& \left(X_{a, h}\left(i^{1-p} u_{a+(-1)^{a_{j-1}} 2^{j-1}+(-1)^{a_{0}}}+\gamma_{r}\left(i^{1-p} u_{a+(-1)^{a_{j-1}} 2^{j-1}+(-1)^{a_{0}}}\right)\right) \otimes v_{h}\right) \\
& \left.-Y_{a, h}\left(i^{-p} u_{a+(-1)^{a_{j-1}} 2^{j-1}+(-1)^{a_{0}}}+\gamma_{r}\left(i^{-p} u_{a+(-1)^{a_{j-1}} 2^{j-1}+(-1)^{a_{0}}}\right)\right) \otimes v_{h}\right)
\end{aligned}
$$

\section{Case $r \equiv 1(\bmod 8)$}

For any point) $Z \in S\left(\tilde{\Delta}_{r} \otimes_{\mathbb{R}} \mathbb{R}^{m}\right)=S^{d_{r} m}$,

$$
Z=\sum_{h=1}^{m} \sum_{a=0}^{2^{[r / 2]-1}-1} X_{a, h}\left(u_{a} \otimes v_{h}\right)+Y_{a, h}\left(i u_{a} \otimes v_{h}\right),
$$

where $\left\{v_{1}, \ldots, v_{m}\right\}$ is an orthonormal basis of $\mathbb{R}^{m}$ and $X_{a, h}, Y_{a, h} \in \mathbb{R}$, there are $r$ - 1 point-wise linearly independent vector fields given as follows. For $p=2$,

$$
\begin{aligned}
& {\left.\left.\left[\kappa_{r}\left(e_{1} e_{2}\right) \otimes \operatorname{Id}_{m}\right]\left(\sum_{h=1}^{m} \sum_{a=0}^{2^{[r / 2]-1}-1} X_{a, h}\left(u_{a}+\gamma_{r}\left(u_{a}\right)\right) \otimes v_{h}\right)+Y_{a, h}\left(i u_{a}+\gamma_{r}\left(i u_{a}\right)\right) \otimes v_{h}\right)\right) } \\
= & \left.\left.\sum_{h=1}^{m} \sum_{a=0}^{2^{[r / 2]-1}-1}(-1)^{a_{0}}\left(-Y_{a, h}\left(u_{a}+\gamma_{r}\left(u_{a}\right)\right) \otimes v_{h}\right)+X_{a, h}\left(i u_{a}+\gamma_{r}\left(i u_{a}\right)\right) \otimes v_{h}\right)\right) .
\end{aligned}
$$

For $3 \leq p \leq r-1$,

$$
\begin{aligned}
& {\left.\left.\left[\kappa_{r}\left(e_{1} e_{p}\right) \otimes \operatorname{Id}_{m}\right]\left(\sum_{h=1}^{m} \sum_{a=0}^{2^{[r / 2]-1}-1} X_{a, h}\left(u_{a}+\gamma_{r}\left(u_{a}\right)\right) \otimes v_{h}\right)+Y_{a, h}\left(i u_{a}+\gamma_{r}\left(i u_{a}\right)\right) \otimes v_{h}\right)\right) } \\
= & \sum_{h=1}^{m} \sum_{a=0}^{2^{[r / 2]-1}-1}(-1)^{2 j-1+\sum_{s=0}^{j-2} a_{s}+a_{j-1}(-2 j+p+1)}\left(X_{a, h}\left(i^{1-p} u_{a+(-1)^{a_{j-1}} 2^{j-1}+(-1)^{a_{0}}}+\gamma_{r}\left(i^{1-p} u_{a+(-1)^{a_{j-1}}} 2^{j-1}+(-1)^{a_{0}}\right)\right) \otimes v_{h}\right)
\end{aligned}
$$




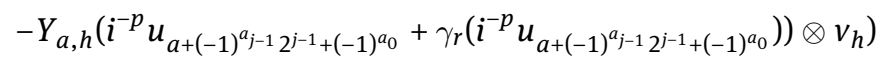

For $p=r$,

$$
\begin{aligned}
& \left.\left.\quad\left[\kappa_{r}\left(e_{1} e_{r}\right) \otimes \mathrm{Id}_{m}\right]\left(\sum_{h=1}^{m} \sum_{a=0}^{2^{[r / 2]-1}-1} X_{a, h}\left(u_{a}+\gamma_{r}\left(u_{a}\right)\right) \otimes v_{h}\right)+Y_{a, h}\left(i u_{a}+\gamma_{r}\left(i u_{a}\right)\right) \otimes v_{h}\right)\right) \\
& =\sum_{h=1}^{m} \sum_{a=0}^{2^{[r / 2]-1}-1}(-1)^{[r / 2]+1+\sum_{l=0}^{[r / 2]-1} a_{l}}\left(X_{a, h}\left(u_{a+(-1)^{a_{0}}}+\gamma_{r}\left(u_{a+(-1)^{a_{0}}}\right)\right) \otimes v_{h}\right) \\
& \left.\quad+Y_{a, h}\left(i u_{a+(-1)^{a_{0}}}+\gamma_{r}\left(i u_{a+(-1)^{a_{0}}}\right)\right) \otimes v_{h}\right) .
\end{aligned}
$$

\section{Case $r \equiv 2,4(\bmod 8)$}

For any point $Z \in S\left(\tilde{\Delta}_{r} \otimes_{\mathbb{R}} \mathbb{R}^{m}\right)=S^{d_{r} m}$ if $r \equiv 2(\bmod 8)\left(\operatorname{resp} . Z \in S\left(\tilde{\Delta}_{r}^{+} \otimes_{\mathbb{R}} \mathbb{R}^{m}\right)=S^{d_{r} m}\right.$ if $\left.r \equiv 4(\bmod 8)\right)$,

$$
Z=\sum_{h=1}^{m} \sum_{\left\{a=0, \ldots, 2^{r / 2}-1, \sum a_{l} \equiv 0(\bmod 2)\right\}} X_{a, h}\left(u_{a} \otimes v_{h}\right)+Y_{a, h}\left(i u_{a} \otimes v_{h}\right),
$$

where $\left\{v_{1}, \ldots, v_{m}\right\}$ is an orthonormal basis of $\mathbb{R}^{m}$ and $X_{a, h}, Y_{a, h} \in \mathbb{R}$, there are $r-1$ point-wise linearly independent vector fields given as follows. For $p=2$,

$$
\begin{aligned}
& {\left[\kappa_{r}\left(e_{1} e_{2}\right) \otimes \operatorname{Id}_{m}\right]\left(\sum_{h=1}^{m} \sum_{\left\{a=0, \ldots, 2^{r / 2}-1, \sum a_{l} \equiv 0(\bmod 2)\right\}} X_{a, h}\left(u_{a} \otimes v_{h}\right)+Y_{a, h}\left(i u_{a} \otimes v_{h}\right)\right) } \\
= & \sum_{h=1}^{m} \sum_{\left\{a=0, \ldots, 2^{r / 2}-1, \sum a_{l} \equiv 0(\bmod 2)\right\}}(-1)^{a_{0}}\left(-Y_{a, h}\left(u_{a} \otimes v_{h}\right)+X_{a, h}\left(i u_{a} \otimes v_{h}\right)\right) .
\end{aligned}
$$

For $3 \leq p \leq r$,

$$
\begin{aligned}
& {\left[\kappa_{r}\left(e_{1} e_{p}\right) \otimes \operatorname{Id}_{m}\right]\left(\sum_{h=1}^{m} \sum_{\left\{a=0, \ldots, 2^{r / 2}-1, \sum a_{l} \equiv 0(\bmod 2)\right\}} X_{a, h}\left(u_{a} \otimes v_{h}\right)+Y_{a, h}\left(i u_{a} \otimes v_{h}\right)\right)}
\end{aligned}
$$

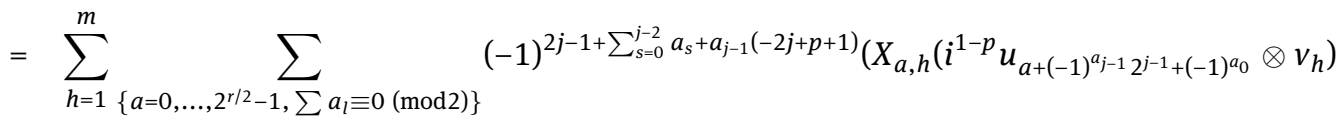

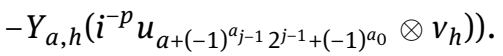

\section{Example: Vector fields on $S^{31}$}

In this subsection, we compute explicitly a maximal set of orthogonal linearly independent vector fields on $S^{31}$. Recall that $\mathrm{Cl}_{10}^{0}$ is the biggest even Clifford algebra with $\mathbb{R}^{32}$ as representation space, so there are 9 linearly independent orthogonal vector fields on the sphere $S^{31}$. Let $Z \in S^{31}$, in terms of our basis

$$
\begin{aligned}
Z:= & \left(X_{0}+i Y_{0}\right) u_{0}+\left(X_{3}+i Y_{3}\right) u_{3}+\left(X_{5}+i Y_{5}\right) u_{5}+\left(X_{6}+i Y_{6}\right) u_{6}+\left(X_{9}+i Y_{9}\right) u_{9}+\left(X_{10}+i Y_{10}\right) u_{10} \\
& +\left(X_{12}+i Y_{12}\right) u_{12}+\left(X_{15}+i Y_{15}\right) u_{15}+\left(X_{17}+i Y_{17}\right) u_{17}+\left(X_{18}+i Y_{18}\right) u_{18}+\left(X_{20}+i Y_{20}\right) u_{20} \\
& +\left(X_{23}+i Y_{23}\right) u_{23}+\left(X_{24}+i Y_{24}\right) u_{24}+\left(X_{27}+i Y_{27}\right) u_{27}+\left(X_{29}+i Y_{29}\right) u_{29}+\left(X_{30}+i Y_{30}\right) u_{30}
\end{aligned}
$$

then a set of linearly independent orthogonal vector fields is given by

$V_{1}=e_{1} e_{2} \cdot Z$ 
$=\left(i X_{0}-Y_{0}\right) u_{0}+\left(-i X_{3}+Y_{3}\right) u_{3}+\left(-i X_{5}+Y_{5}\right) u_{5}+\left(i X_{6}-Y_{6}\right) u_{6}+\left(-i X_{9}+Y_{9}\right) u_{9}+\left(i X_{10}-Y_{10}\right) u_{10}$ $+\left(i X_{12}-Y_{12}\right) u_{12}+\left(-i X_{15}+Y_{15}\right) u_{15}+\left(-i X_{17}+Y_{17}\right) u_{17}+\left(i X_{18}-Y_{18}\right) u_{18}+\left(i X_{20}-Y_{20}\right) u_{20}$ $+\left(-i X_{23}+Y_{23}\right) u_{23}+\left(i X_{24}-Y_{24}\right) u_{24}+\left(-i X_{27}+Y_{27}\right) u_{27}+\left(-i X_{29}+Y_{29}\right) u_{29}+\left(i X_{30}-Y_{30}\right) u_{30}$

$V_{2}=e_{1} e_{3} \cdot Z$

$=\left(-X_{3}-i Y_{3}\right) u_{0}+\left(X_{0}+i Y_{0}\right) u_{3}+\left(X_{6}+i Y_{6}\right) u_{5}+\left(-X_{5}-i Y_{5}\right) u_{6}+\left(X_{10}+i Y_{10}\right) u_{9}+\left(-X_{9}-i Y_{9}\right) u_{10}$ $+\left(-X_{15}-i Y_{15}\right) u_{12}+\left(X_{12}+i Y_{12}\right) u_{15}+\left(X_{18}+i Y_{18}\right) u_{17}+\left(-X_{17}-i Y_{17}\right) u_{18}+\left(-X_{23}-i Y_{23}\right) u_{20}$ $+\left(X_{20}+i Y_{20}\right) u_{23}+\left(-X_{27}-i Y_{27}\right) u_{24}+\left(X_{24}+i Y_{24}\right) u_{27}+\left(X_{30}+i Y_{30}\right) u_{29}+\left(-X_{29}-i Y_{29}\right) u_{30}$

$V_{3}=e_{1} e_{4} \cdot Z$

$=\left(-i X_{3}+Y_{3}\right) u_{0}+\left(-i X_{0}+Y_{0}\right) u_{3}+\left(i X_{6}-Y_{6}\right) u_{5}+\left(i X_{5}-Y_{5}\right) u_{6}+\left(i X_{10}-Y_{10}\right) u_{9}+\left(i X_{9}-Y_{9}\right) u_{10}$ $+\left(-i X_{15}+Y_{15}\right) u_{12}+\left(-i X_{12}+Y_{12}\right) u_{15}+\left(i X_{18}-Y_{18}\right) u_{17}+\left(i X_{17}-Y_{17}\right) u_{18}+\left(-i X_{23}+Y_{23}\right) u_{20}$ $+\left(-i X_{20}+Y_{20}\right) u_{23}+\left(-i X_{27}+Y_{27}\right) u_{24}+\left(-i X_{24}+Y_{24}\right) u_{27}+\left(i X_{30}-Y_{30}\right) u_{29}+\left(i X_{29}-Y_{29}\right) u_{30}$

$V_{4}=e_{1} e_{5} \cdot Z$

$=\left(X_{5}+i Y_{5}\right) u_{0}+\left(X_{6}+i Y_{6}\right) u_{3}+\left(-X_{0}-i Y_{0}\right) u_{5}+\left(-X_{3}-i Y_{3}\right) u_{6}+\left(-X_{12}-i Y_{12}\right) u_{9}+\left(-X_{15}-i Y_{15}\right) u_{10}$ $+\left(X_{9}+i Y_{9}\right) u_{12}+\left(X_{10}+i Y_{10}\right) u_{15}+\left(-X_{20}-i Y_{20}\right) u_{17}+\left(-X_{23}-i Y_{23}\right) u_{18}+\left(X_{17}+i Y_{17}\right) u_{20}$ $+\left(X_{18}+i Y_{18}\right) u_{23}+\left(X_{29}+i Y_{29}\right) u_{24}+\left(X_{30}+i Y_{30}\right) u_{27}+\left(-X_{24}-i Y_{24}\right) u_{29}+\left(-X_{27}-i Y_{27}\right) u_{30}$

$V_{5}=e_{1} e_{6} \cdot Z$

$=\left(i X_{5}-Y_{5}\right) u_{0}+\left(i X_{6}-Y_{6}\right) u_{3}+\left(i X_{0}-Y_{0}\right) u_{5}+\left(i X_{3}-Y_{3}\right) u_{6}+\left(-i X_{12}+Y_{12}\right) u_{9}+\left(-i X_{15}+Y_{15}\right) u_{10}$ $+\left(-i X_{9}+Y_{9}\right) u_{12}+\left(-i X_{10}+Y_{10}\right) u_{15}+\left(-i X_{20}+Y_{20}\right) u_{17}+\left(-i X_{23}+Y_{23}\right) u_{18}+\left(-i X_{17}+Y_{17}\right) u_{20}$ $+\left(-i X_{18}+Y_{18}\right) u_{23}+\left(i X_{29}-Y_{29}\right) u_{24}+\left(i X_{30}-Y_{30}\right) u_{27}+\left(i X_{24}-Y_{24}\right) u_{29}+\left(i X_{27}-Y_{27}\right) u_{30}$

$V_{6}=e_{1} e_{7} \cdot Z$

$=\left(-X_{9}-i Y_{9}\right) u_{0}+\left(-X_{10}-i Y_{10}\right) u_{3}+\left(-X_{12}-i Y_{12}\right) u_{5}+\left(-X_{15}-i Y_{15}\right) u_{6}+\left(X_{0}+i Y_{0}\right) u_{9}+\left(X_{3}+i Y_{3}\right) u_{10}$ $+\left(X_{5}+i Y_{5}\right) u_{12}+\left(X_{6}+i Y_{6}\right) u_{15}+\left(X_{24}+i Y_{24}\right) u_{17}+\left(X_{27}+i Y_{27}\right) u_{18}+\left(X_{29}+i Y_{29}\right) u_{20}$ $+\left(X_{30}+i Y_{30}\right) u_{23}+\left(-X_{17}-i Y_{17}\right) u_{24}+\left(-X_{18}-i Y_{18}\right) u_{27}+\left(-X_{20}-i Y_{20}\right) u_{29}+\left(-X_{23}-i Y_{23}\right) u_{30}$

$V_{7}=e_{1} e_{8} \cdot Z$

$=\left(-i X_{9}+Y_{9}\right) u_{0}+\left(-i X_{10}+Y_{10}\right) u_{3}+\left(-i X_{12}+Y_{12}\right) u_{5}+\left(-i X_{15}+Y_{15}\right) u_{6}+\left(-i X_{0}+Y_{0}\right) u_{9}+\left(-i X_{3}+Y_{3}\right) u_{10}$ $+\left(-i X_{5}+Y_{5}\right) u_{12}+\left(-i X_{6}+Y_{6}\right) u_{15}+\left(i X_{24}-Y_{24}\right) u_{17}+\left(i X_{27}-Y_{27}\right) u_{18}+\left(i X_{29}-Y_{29}\right) u_{20}$ $+\left(i X_{30}-Y_{30}\right) u_{23}+\left(i X_{17}-Y_{17}\right) u_{24}+\left(i X_{18}-Y_{18}\right) u_{27}+\left(i X_{20}-Y_{20}\right) u_{29}+\left(i X_{23}-Y_{23}\right) u_{30}$

$V_{8}=e_{1} e_{9} \cdot Z$

$=\left(X_{17}+i Y_{17}\right) u_{0}+\left(X_{18}+i Y_{18}\right) u_{3}+\left(X_{20}+i Y_{20}\right) u_{5}+\left(X_{23}+i Y_{23}\right) u_{6}+\left(X_{24}+i Y_{24}\right) u_{9}+\left(X_{27}+i Y_{27}\right) u_{10}$ $+\left(X_{29}+i Y_{29}\right) u_{12}+\left(X_{30}+i Y_{30}\right) u_{15}+\left(-X_{0}-i Y_{0}\right) u_{17}+\left(-X_{3}-i Y_{3}\right) u_{18}+\left(-X_{5}-i Y_{5}\right) u_{20}$ $+\left(-X_{6}-i Y_{6}\right) u_{23}+\left(-X_{9}-i Y_{9}\right) u_{24}+\left(-X_{10}-i Y_{10}\right) u_{27}+\left(-X_{12}-i Y_{12}\right) u_{29}+\left(-X_{15}-i Y_{15}\right) u_{30}$

$V_{9}=e_{1} e_{10} \cdot Z$

$=\left(i X_{17}-Y_{17}\right) u_{0}+\left(i X_{18}-Y_{18}\right) u_{3}+\left(i X_{20}-Y_{20}\right) u_{5}+\left(i X_{23}-Y_{23}\right) u_{6}+\left(i X_{24}-Y_{24}\right) u_{9}+\left(i X_{27}-Y_{27}\right) u_{10}$ $+\left(i X_{29}-Y_{29}\right) u_{12}+\left(i X_{30}-Y_{30}\right) u_{15}+\left(i X_{0}-Y_{0}\right) u_{17}+\left(i X_{3}-Y_{3}\right) u_{18}+\left(i X_{5}-Y_{5}\right) u_{20}$ $+\left(i X_{6}-Y_{6}\right) u_{23}+\left(i X_{9}-Y_{9}\right) u_{24}+\left(i X_{10}-Y_{10}\right) u_{27}+\left(i X_{12}-Y_{12}\right) u_{29}+\left(i X_{15}-Y_{15}\right) u_{30}$.

In terms of coordinate vectors, one can write these vector fields as follows

$$
\begin{aligned}
V_{1}= & \left(-Y_{0}, X_{0}, Y_{3},-X_{3}, Y_{5},-X_{5},-Y_{6}, X_{6}, Y_{9},-X_{9},-Y_{10}, X_{10},-Y_{12}, X_{12}, Y_{15},\right. \\
& \left.-X_{15}, Y_{17},-X_{17},-Y_{18}, X_{18},-Y_{20}, X_{20}, Y_{23},-X_{23},-Y_{24}, X_{24}, Y_{27},-X_{27}, Y_{29},-X_{29},-Y_{30}, X_{30}\right), \\
V_{2}= & \left(-X_{3},-Y_{3}, X_{0}, Y_{0}, X_{6}, Y_{6},-X_{5},-Y_{5}, X_{10}, Y_{10},-X_{9},-Y_{9},-X_{15},-Y_{15}, X_{12}, Y_{12},\right. \\
& \left.X_{18}, Y_{18},-X_{17},-Y_{17},-X_{23},-Y_{23}, X_{20}, Y_{20},-X_{27},-Y_{27}, X_{24}, Y_{24}, X_{30}, Y_{30},-X_{29},-Y_{29}\right), \\
V_{3}= & \left(Y_{3},-X_{3}, Y_{0},-X_{0},-Y_{6}, X_{6},-Y_{5}, X_{5},-Y_{10}, X_{10},-Y_{9}, X_{9}, Y_{15},-X_{15}, Y_{12},-X_{12},\right.
\end{aligned}
$$




$$
\begin{aligned}
& \left.-Y_{18}, X_{18},-Y_{17}, X_{17}, Y_{23},-X_{23}, Y_{20},-X_{20}, Y_{27},-X_{27}, Y_{24},-X_{24},-Y_{30}, X_{30},-Y_{29}, X_{29}\right), \\
V_{4}= & \left(X_{5}, Y_{5}, X_{6}, Y_{6},-X_{0},-Y_{0},-X_{3},-Y_{3},-X_{12},-Y_{12},-X_{15},-Y_{15}, X_{9}, Y_{9}, X_{10}, Y_{10},\right. \\
& \left.-X_{20},-Y_{20},-X_{23},-Y_{23}, X_{17}, Y_{17}, X_{18}, Y_{18}, X_{29}, Y_{29}, X_{30}, Y_{30},-X_{24},-Y_{24},-X_{27},-Y_{27}\right), \\
V_{5}= & \left(-Y_{5}, X_{5},-Y_{6}, X_{6},-Y_{0},-X_{0},-Y_{3}, X_{3}, Y_{12},-X_{12}, Y_{15},-X_{15}, Y_{9},-X_{9}, Y_{10},-X_{10},\right. \\
& \left.\left.Y_{20},-X_{20}, Y_{23},-X_{23}, Y_{17},-X_{17}, Y_{18},-X_{18},-Y_{29}, X_{29},-Y_{30}, X_{30},-Y_{24}, X_{24},-Y_{27}, X_{27}\right)\right), \\
V_{6}= & \left(-X_{9},-Y_{9},-X_{10},-Y_{10},-X_{12},-Y_{12},-X_{15}, Y_{15}, X_{0}, Y_{0}, X_{3}, Y_{3}, X_{5}, Y_{5}, X_{6}, Y_{6},\right. \\
& \left.X_{24}, Y_{24}, X_{27}, Y_{27}, X_{29}, Y_{29}, X_{30}, Y_{30},-X_{17},-Y_{17},-X_{18},-Y_{18},-X_{20},-Y_{20},-X_{23},-Y_{23}\right), \\
V_{7}= & \left(Y_{9},-X_{9}, Y_{10},-X_{10}, Y_{12},-X_{12}, Y_{15},-X_{15}, Y_{0},-X_{0}, Y_{3},-X_{3}, Y_{5},-X_{5}, Y_{6},-X_{6},\right. \\
& \left.-Y_{24}, X_{24},-Y_{27}, X_{27},-Y_{29}, X_{29},-Y_{30}, X_{30},-Y_{17}, X_{17},-Y_{18}, X_{18},-Y_{20}, X_{20},-Y_{23}, X_{23}\right), \\
V_{8}= & \left(X_{17}, Y_{17}, X_{18}, Y_{18}, X_{20}, Y_{20}, X_{23}, Y_{23}, X_{24}, Y_{24}, X_{27}, Y_{27}, X_{29}, Y_{29}, X_{30}, Y_{30},\right. \\
& \left.-X_{0},-Y_{0},-X_{3},-Y_{3},-X_{5},-Y_{5},-X_{6},-Y_{6},-X_{9},-Y_{9},-X_{10},-Y_{10},-X_{12},-Y_{12},-X_{15},-Y_{15}\right), \\
V_{9}, & \left(-Y_{17}, X_{17},-Y_{18}, X_{18},-Y_{20}, X_{20},-Y_{23}, X_{23},-Y_{24}, X_{24},-Y_{27}, X_{27},-Y_{29}, X_{29},-Y_{30}, X_{30},\right. \\
& \left.-Y_{0}, X_{0},-Y_{3}, X_{3},-Y_{5}, X_{5},-Y_{6}, X_{6},-Y_{9}, X_{9},-Y_{10}, X_{10},-Y_{12}, X_{12},-Y_{15}, X_{15}\right) .
\end{aligned}
$$

Relabelling the entries of $Z$,

$$
\begin{aligned}
Z:= & \left(v_{1}, v_{2}, v_{3}, v_{4}, v_{5}, v_{6}, v_{7}, v_{8}, v_{9}, v_{10}, v_{11}, v_{12}, v_{13}, v_{14}, v_{15}, v_{16},\right. \\
& \left.v_{17}, v_{18}, v_{19}, v_{20}, v_{21}, v_{22}, v_{23}, v_{24}, v_{25}, v_{26}, v_{27}, v_{28}, v_{29}, v_{30}, v_{31}, v_{32}\right),
\end{aligned}
$$

the vector fields are the following

$$
\begin{aligned}
& V_{1}=\left(-v_{2}, v_{1}, v_{4},-v_{3}, v_{6},-v_{5},-v_{8}, v_{7}, v_{10},-v_{9},-v_{12}, v_{11},-v_{14}, v_{13}, v_{16},-v_{15},\right. \\
& \left.v_{18},-v_{17},-v_{20}, v_{19},-v_{22}, v_{21}, v_{24},-v_{23},-v_{26}, v_{25}, v_{28},-v_{27}, v_{30},-v_{29},-v_{32}, v_{31}\right) \text {, } \\
& V_{2}=\left(-v_{3},-v_{4}, v_{1}, v_{2}, v_{7}, v_{8},-v_{5},-v_{6}, v_{11}, v_{12},-v_{9},-v_{10},-v_{15},-v_{16}, v_{13}, v_{14}\right. \text {, } \\
& \left.v_{19}, v_{20},-v_{17},-v_{18},-v_{23},-v_{24}, v_{21}, v_{22},-v_{27},-v_{28}, v_{25}, v_{26}, v_{31}, v_{32},-v_{29},-v_{30}\right) \text {, } \\
& V_{3}=\left(v_{4},-v_{3}, v_{2},-v_{1},-v_{8}, v_{7},-v_{6}, v_{5},-v_{12}, v_{11},-v_{10}, v_{9}, v_{16},-v_{15}, v_{14},-v_{13}\right. \text {, } \\
& \left.-v_{20}, v_{19},-v_{18}, v_{17}, v_{24},-v_{23}, v_{22},-v_{21}, v_{28},-v_{27}, v_{26},-v_{25},-v_{32}, v_{31},-v_{30}, v_{29}\right) \text {, } \\
& V_{4}=\left(v_{5}, v_{6}, v_{7}, v_{8},-v_{1},-v_{2},-v_{3},-v_{4},-v_{13},-v_{14},-v_{15},-v_{16}, v_{9}, v_{10}, v_{11}, v_{12}\right. \text {, } \\
& \left.-v_{21},-v_{22},-v_{23},-v_{24}, v_{17}, v_{18}, v_{19}, v_{20}, v_{29}, v_{30}, v_{31}, v_{32},-v_{25},-v_{26},-v_{27},-v_{28}\right) \text {, } \\
& V_{5}=\left(-v_{6}, v_{5},-v_{8}, v_{7},-v_{2},-v_{1},-v_{4}, v_{3}, v_{14},-v_{13}, v_{16},-v_{15}, v_{10},-v_{9}, v_{12},-v_{11}\right. \text {, } \\
& \left.v_{22},-v_{21}, v_{24},-v_{23}, v_{18},-v_{17}, v_{20},-v_{19},-v_{30}, v_{29},-v_{32}, v_{31},-v_{26}, v_{25},-v_{28}, v_{27}\right) \text {, } \\
& V_{6}=\left(-v_{9},-v_{10},-v_{11},-v_{12},-v_{13},-v_{14},-v_{15}, v_{16}, v_{1}, v_{2}, v_{3}, v_{4}, v_{5}, v_{6}, v_{7}, v_{8}\right. \text {, } \\
& \left.v_{25}, v_{26}, v_{27}, v_{28}, v_{29}, v_{30}, v_{31}, v_{32},-v_{17},-v_{18},-v_{19},-v_{20},-v_{21},-v_{22},-v_{23},-v_{24}\right) \text {, } \\
& V_{7}=\left(v_{10},-v_{9}, v_{12},-v_{11}, v_{14},-v_{13}, v_{16},-v_{15}, v_{2},-v_{1}, v_{4},-v_{3}, v_{6},-v_{5}, v_{8},-v_{7}\right. \text {, } \\
& \left.-v_{26}, v_{25},-v_{28}, v_{27},-v_{30}, v_{29},-v_{32}, v_{31},-v_{18}, v_{17},-v_{20}, v_{19},-v_{22}, v_{21},-v_{24}, v_{23}\right) \text {, } \\
& V_{8}=\left(v_{17}, v_{18}, v_{19}, v_{20}, v_{21}, v_{22}, v_{23}, v_{24}, v_{25}, v_{26}, v_{27}, v_{28}, v_{29}, v_{30}, v_{31}, v_{32}\right. \text {, } \\
& \left.-v_{1},-v_{2},-v_{3},-v_{4},-v_{5},-v_{6},-v_{7},-v_{8},-v_{9},-v_{10},-v_{11},-v_{12},-v_{13},-v_{14},-v_{15},-v_{16}\right) \text {, } \\
& V_{9}=\left(-v_{18}, v_{17},-v_{20}, v_{19},-v_{22}, v_{21},-v_{24}, v_{23},-v_{26}, v_{25},-v_{28}, v_{27},-v_{30}, v_{29},-v_{32}, v_{31}\right. \text {, } \\
& \left.-v_{2}, v_{1},-v_{4}, v_{3},-v_{6}, v_{5},-v_{8}, v_{7},-v_{10}, v_{9},-v_{12}, v_{11},-v_{14}, v_{13},-v_{16}, v_{15}\right) \text {. }
\end{aligned}
$$

Acknowledgement: Both authors were partially supported by CONACyT grant 256126.

\section{References}

[1] Adams J. F.: Lectures on Exceptional Lie Groups, eds. Zafer Mahmud and Mamoru Mimira, University of Chicago Press, Chicago, 1996. 
[2] Adams J. F.: Vector fields of spheres. Ann. of Math. 75 (1962), 603-632.

[3] Arizmendi, G.; Herrera, R.: Journal of Geometry and Physics 97, 77-92.

[4] Baez, J.C.: The octonions. Bull. Amer. Math. Soc. 39 (2002), 145-205.

[5] Baum, H.; Friedrich, T.; Grunewald, R.; Kath, I.: Twistor and Killing spinors on Riemannian manifolds. Seminarberichte [Seminar Reports], 108. Humboldt Universität, Sektion Mathematik, Berlin, 1990. 179 pp.

[6] Brauer, R.; Weyl, H.: American Journal of Mathematics 57, No. 2 (Apr., 1935), pp. 425-449

[7] Budinich, M.: On Clifford algebras and binary integers. (English summary) Adv. Appl. Clifford Algebr. 27 (2017), no. 2, 10071017

[8] Budinich, P.; Trautman, A.: Fock space description of simple spinors Journal of Mathematical Physics 30, 2125 (1989)

[9] Cartan, E.: Le principe de dualitét la théorie des groupes simples et semi-simples. Bulletin sc. Math. (2) 49, 361-374 (1925).

[10] Cartan, E.: The theory of spinors. The M.I.T. Press, Cambridge, Mass. 1967 viii 157 pp. 53.38

[11] Friedrich T. ; Sulanke. S.: Ein Kriterium für die formale Selbstadjungiertheit des Dirac-Operators. Coll. Math. vol. XL, Fasc. 2 (1979), 239-247

[12] Friedrich, T.: Dirac operators in Riemannian geometry. Translated from the 1997 German original by Andreas Nestke. Graduate Studies in Mathematics, 25. American Mathematical Society, Providence, RI, 2000. xvi+195 pp. ISBN: 0-8218-2055-9.

[13] Harvey, F. R.: Spinors and calibrations. Perspectives in Mathematics, 9. Academic Press, Inc., Boston, MA, 1990. xiv+323 pp.

[14] Husemoller, D.: Fibre bundles. Third edition. Graduate Texts in Mathematics, 20. Springer-Verlag, New York, 1994. xx+353 pp. ISBN: 0-387-94087-1

[15] A. Hurwitz, A.: Uber die Komposition der quadratischen Formen, Math. Ann. 88 (1922) 1-25., reproduced in Mathematische Werke: Zahlentheorie, Algebra und Geometrie,.Band II, Birkhäuser (1963), 641-666.

[16] Lawson, H. B., Jr.; Michelsohn, M.-L.: Spin geometry. Princeton Mathematical Series, 38. Princeton University Press, Princeton, NJ, 1989. xii+427 pp. ISBN: 0-691-08542-0

[17] Lounesto, P.: Octonions and triality. Adv. Appl. Clifford Algebras 11 (2001), no. 2, 191-213 (2002)

[18] Lounesto, P.: Clifford algebras and spinors. London Mathematical Society Lecture Note Series, 239. Cambridge University Press, Cambridge, 1997. x+306 pp. ISBN: 0-521-59916-4

[19] Ognikyan, A.A.: Combinatorial Construction of Tangent Vector Fields on Spheres, Math. Notes 83 (2008) 590-605.

[20] Parton, M.; Piccinni, P.: Spheres with more than 7 vector fields: All the fault of Spin(9). Linear Algebra and its Applications 438 (2013) 1113-1131. 